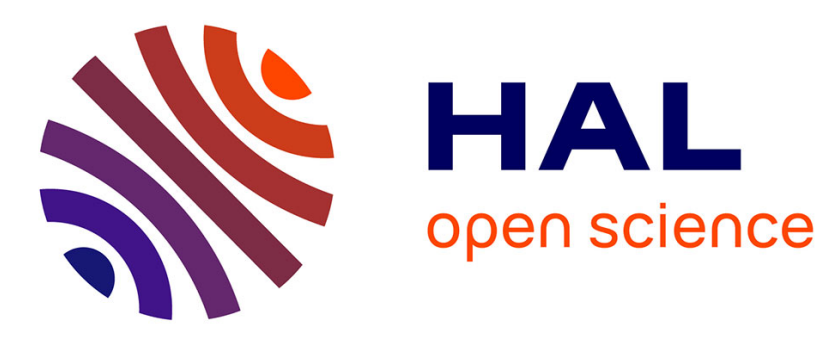

\title{
Circular Dichroism in Molecular Frame Photoemission
}

Danielle Dowek

\section{To cite this version:}

Danielle Dowek. Circular Dichroism in Molecular Frame Photoemission. Molecular Physics, 2007, 105

(11-12), pp.1757-1768. 10.1080/00268970701501802 . hal-00513120

\section{HAL Id: hal-00513120 \\ https://hal.science/hal-00513120}

Submitted on 1 Sep 2010

HAL is a multi-disciplinary open access archive for the deposit and dissemination of scientific research documents, whether they are published or not. The documents may come from teaching and research institutions in France or abroad, or from public or private research centers.
L'archive ouverte pluridisciplinaire HAL, est destinée au dépôt et à la diffusion de documents scientifiques de niveau recherche, publiés ou non, émanant des établissements d'enseignement et de recherche français ou étrangers, des laboratoires publics ou privés. 


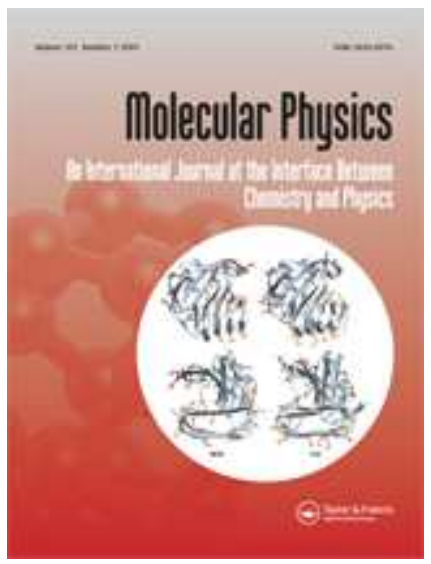

\section{Circular Dichroism in Molecular Frame Photoemission}

\begin{tabular}{|r|l|}
\hline Journal: & Molecular Physics \\
\hline Manuscript ID: & TMPH-2007-0086.R1 \\
\hline Manuscript Type: & Full Paper \\
\hline Date Submitted by the \\
Author: & 28-May-2007 \\
\hline Complete List of Authors: & DOWEK, Danielle; LCAM, Université Paris-Sud \\
\hline Keywords: & $\begin{array}{l}\text { Photoionization of molecules, Circular dichroism, Molecular frame } \\
\text { photoemission }\end{array}$ \\
\hline
\end{tabular}

\section{ScholarONE" \\ Manuscript Central}


1

\section{Author list}

\section{Danielle DOWEK}

Laboratoire des Collisions Atomiques et Moléculaires (LCAM, UMR Université Paris Sud et CNRS, No. 8625),

Bâtiment 351, Université Paris Sud, F-91405 Orsay Cedex,

FRANCE

e-mail: danielle.dowek@u-psud.fr

Phone: 33-1-6915 7672

FAX : 33-1-6915 7671

\section{Mogens LEBECH}

The Photon Factory, Institute of Materials Structure Science,

Department: High Energy Accelerator Research Organization

1-1 Oho Tsukuba-shi

Ibaraki-ken 305-0801,

JAPAN

e-mail: mogenslebech@yahoo.dk

Phone: +81-29-864-5699

Jean-Christophe HOUVER

Laboratoire des Collisions Atomiques et Moléculaires (LCAM, UMR Université Paris Sud et CNRS, No. 8625),

Bâtiment 351, Université Paris Sud, F-91405 Orsay Cedex,

FRANCE

| e-mail: jean-christophe.houver@wanadoo.fr Phone: 33-1-6915 7673

FAX : 33-1-6915 7671

Robert R. LUCCHESE

Department of Chemistry, Texas A\&M University, College Station, Texas 77843,

USA

e-mail: lucchese @ mail.chem.tamu.edu

Phone: 1979 845-0187

Fax: $1979845-4719$

\section{Key words}

Photoionization of molecules; Circular dichroism, Molecular frame photoemission; 


\title{
Circular Dichroism in Molecular Frame Photoemission
}

\author{
D. Dowek, M. Lebech ${ }^{\S}$ J. C. Houver \\ Laboratoire des Collisions Atomiques et Moléculaires (LCAM, UMR Université Paris Sud et \\ CNRS, No. 8625), Bâtiment 351, Université Paris Sud, F-91405 Orsay Cedex, France
}

Robert R. Lucchese

Department of Chemistry, Texas A\&M University, College Station, Texas 77843

\footnotetext{
${ }^{\S}$ Present address : The Photon Factory, Institute of Materials Structure Science, 1-1 Oho Tsukuba-shi Ibaraki-ken 305-0801, JAPAN
} 
Deleted: describing the zero-energy phase shifts

\begin{abstract}
We report a comparative experimental and theoretical study of circular dichroism in electron angular distribution (CDAD) in the molecular frame (MF) of linear molecules photoionized by circularly or elliptically polarized light. The CDAD is derived from the analysis of the complete molecular frame angular distribution (MFPAD) $I\left(\chi, \theta_{e}, \phi_{e}\right)$, where $\chi$ is the orientation of the molecule with respect to the light propagation axis and $\left(\theta_{e}, \phi_{e}\right)$ the electron emission direction in the MF, using the vector correlation method. The CDAD is quantified by the $\theta_{e}$ dependence of the left-right emission asymmetry maximum in the plane perpendicular to the light propagation axis $\mathbf{k}$, for a space fixed molecule orthogonal to $\mathbf{k}$. The experimental results for selected valence shell photoionization (PI) reactions in $\mathrm{NO}, \mathrm{O}_{2}, \mathrm{~N}_{2} \mathrm{O}$ compare very well with the multichannel Schwinger configuration interaction (MCSCI) $a b$ initio calculations. Combined with a simple model of the ionization process, including the partial-wave composition of the initial state and phase shifts estimated from quantum defects for the various scattering partial-waves, these results provide the basis for a general discussion of the circular dichroism effect. This study enables us to disentangle the influence of the ( $s p \sigma$, $p \pi \ldots)$ initial valence shell ionized orbital and that of the scattering dynamics on some fingerprint properties of the CDAD. On the other hand, the $\mathrm{CDAD}$ for $s \sigma \mathrm{K}$ shell is purely assigned to a final state scattering effect. This analysis will be extended to PI of non-linear molecules where the circular dichroism characterizes recoil frame photoelectron angular distributions (RFPADs).
\end{abstract}




\section{Introduction}

Angle resolved molecular frame photoemission constitutes a powerful means to investigate the photoionization dynamics of small molecules, and has lead to several developments in the recent years [1]. In particular, the molecular frame photoelectron angular distributions (MFPADs) determined for photoionization (PI) induced by circularly or elliptically polarized light give access to the most complete information in terms of the dipole matrix elements of the transition, their magnitudes and phase shifts [2-6]. These transition moments depend on two main factors: the initial and final bound states in the PI process, and the photoelectron scattering dynamics. In this paper we focus on some qualitative features of circular dichroism in molecular frame photoemission, and we discuss the possibility of disentangling the role of the spectroscopic properties of the initial and final bound states from that of the electron scattering dynamics in determining the magnitude of the circular dichroism effect.

In the most general sense, circular dichroism $(C D)$ refers to the selective response of a system interacting with right or left handed circularly polarized light, and it reflects the existence of the chiral character of the system. The CD in total photoabsorption cross sections of chiral molecules having no plane or center of symmetry has been known since the work of Pasteur [7]: it results from an interference between electric and magnetic dipole terms, or electric dipole-electric quadrupole terms and therefore the effects are small $\left(<10^{-3}\right)$ relative to the total cross section. Until very recently [8], circular dichroism spectroscopy has then been dedicated to the characterization of liquid phase samples [9]. However, for photoelectron angular-resolved observables achievable in gas phase photoionization (PI) of molecules, two kinds of $\mathrm{CD}$ effects relying on the handedness of the system composed of the incoming photon and target [10] were predicted in the simple electric dipole approximation, giving rise to a significantly larger Circular Dichroism in the Angular Distribution (CDAD). 
The description of circular dichroism in the electric dipole approximation characterizing laboratory frame differential PI cross section $\sigma(\theta)$ for randomly oriented chiral molecules, where $\theta$ is the photoelectron polar angle referred to the light propagation axis, was derived about three decades ago $[11,12]$. It is characterized by a forward-backward electron ejection asymmetry of the analytical form $D P_{1}(\cos \theta)$, quantified by a dichroism parameter $D$. Realistic numerical calculations recently found that the expected asymmetry may be of the order of $10 \%$ for specific chiral molecules [13]. This prediction lead to the first valence shell [14-17] and K-shell [18] PI observations demonstrating indeed a CDAD, also referred to as photoelectron circular dichroism (PECD), of the order of few percents, reaching 10-20\% for e.g. camphor enantiomers [17]. For core shell ionization, where the localized initial orbital is achiral, the PECD is mainly ascribed to a final state scattering effect [19], whereas in valence shell ionization a significant influence of the initial orbital has been observed [17,19].

The expression of the circular dichroism in the angular distribution for oriented achiral molecules, in the electric-dipole approximation, has been derived by Cherepkov [12]. There the handedness of the system is induced by the spatial non-coplanarity of three vectors, namely the light propagation axis $\mathbf{k}$, the photoelectron momentum $\mathbf{k}_{\mathbf{e}}$, and the molecular axis n. Initially suggested and observed for molecules oriented in a fixed direction of space by adsorption at the surface of a substrate [20,21], CDAD in PI of achiral molecules in the gas phase was first reported in rotationally resolved photoelectron angular distribution [2], for molecules aligned by multiphoton absorption. The CDAD observation for randomly oriented achiral molecules was further demonstrated by recording molecular frame photoelectron angular distributions (MFPADs), taking advantage of dissociative photoionization (DPI) induced by X/VUV one-photon absorption of circularly polarized synchrotron radiation for inner shell $[3,4,22,23]$ and outer shell $[5,6,24,25]$ photoionization. The triplet $\left(\mathbf{k}_{\mathbf{e}}, \mathbf{n}, \mathbf{k}\right)$ is then identified by measuring in coincidence the initial velocity vectors of the photoion and photoelectron for each DPI event. 
In our first investigation of molecular frame photoemission circular dichroism using the vector correlation method [6], we reported the general form of the $\mathrm{I}\left(\chi, \theta_{e}, \phi_{e}\right)$ complete angular distribution for PI of linear molecules, in the dipole approximation as:

$$
\begin{aligned}
I\left(\theta_{e}, \phi_{e}, \chi\right)= & F_{00}\left(\theta_{e}\right)-s_{3} F_{11}\left(\theta_{e}\right) P_{1}^{1}(\cos \chi) \sin \left(\phi_{e}\right)-\frac{1}{2} F_{20}\left(\theta_{e}\right) P_{2}^{0}(\cos \chi) \\
& -\frac{1}{2} F_{21}\left(\theta_{e}\right) P_{2}^{1}(\cos \chi) \cos \left(\phi_{e}\right)-\frac{1}{2} F_{22}\left(\theta_{e}\right) P_{2}^{2}(\cos \chi) \cos 2\left(\phi_{e}\right)
\end{aligned}
$$

where $\chi$ is the polar angle referring the orientation of the molecular axis $\mathbf{n}$ with respect to the light propagation axis $\mathbf{k}$, and $\left(\theta_{\mathrm{e}}, \phi_{\mathrm{e}}\right)$ characterizes the electron emission direction $\underline{\mathbf{k}}_{\mathrm{e}} \_$in the molecular frame defined by the molecular axis and the light propagation axis, as shown in Figure 1. The Stokes parameter $s_{3}$ is equal to -1 for left handed circularly polarized (LHC) light of positive helicity $(h=1)$. In this expression the dependence on $\phi_{e}$ and $\chi$ is through low order analytical functions: the complete dynamical information about the PI reaction is described by the five one-dimensional $F_{L N}\left(\theta_{e}\right)$ functions, which give access to the transition moments between the ionized orbitals and the photoelectron continuum state, their magnitudes and relative phases. The value of $F_{11}$ governs the circular dichroism and gives access to the sign of the phase shifts of the dipole matrix elements. The most favorable geometry for the observation of circular dichroism in the molecular frame is obtained for a molecule oriented perpendicular to the light propagation axis. The CDAD is then characterized by the left-right asymmetry of photoelectron emission which is maximum in the plane perpendicular to $\mathbf{k}\left(\phi_{e}=90^{\circ}\right.$ and $\left.\phi_{e}=270^{\circ}\right)$ as described by the $\sin \left(\phi_{e}\right)$ dependence in equation 1 . We defined the dimensionless CDAD parameter in two equivalent ways, and established an expression in terms of the $F_{L N}\left(\theta_{e}\right)$ functions [6] as follows:

$$
\operatorname{CDAD}_{\left(\chi=90, \phi_{e}=90\right)}=\frac{I_{+1}-I_{-1}}{I_{+1}+I_{-1}}=\operatorname{CDAD}_{(\chi=90, h=+1)}=\frac{I_{90}-I_{270}}{I_{90}+I_{270}}=\frac{2 F_{11}}{2 F_{00}+\frac{1}{2} F_{20}+3 F_{22}}
$$


In our previous studies we have reported observations of molecular frame CDADs for selected valence shell PI reactions for diatomic or linear polyatomic molecules $[6,24,25]$, and more recently for a few $\mathrm{K}$ shell ionization reactions $[22,23]$. The measured values were found to be in fairly good agreement with the calculations using the multichannel Schwinger configuration interaction method (MCSCI), however no general discussion of the circular dichroism has been presented so far. In this paper we report new experimental and theoretical results for two processes, PI of the $\mathrm{N}_{2} \mathrm{O}$ molecule into the $C^{2} \Sigma^{+}(6 \sigma)^{-1}$ state of $\mathrm{N}_{2} \mathrm{O}^{+}$and PI of $\mathrm{NO}$ into the $\mathrm{NO}^{+} \mathrm{B}$ state located in the region $23-24 \mathrm{eV}$ above the $\mathrm{NO}(\mathrm{X}, \mathrm{v}=0)$ ground state, whose symmetry has not been definitely assigned [26-29]. The detailed analysis of these results, together with those obtained earlier for a series of valence shell PI reactions corresponding to ionization of a $s p \sigma$ and a $p \pi$ valence molecular orbital, respectively, point out common characteristics which demonstrate the significant influence of the initial state on the CDAD. The discussion also relies on the comparison of the measured and computed CDADs with the predictions of a simple model for molecular frame photoemission, developed earlier to explore the qualitative features of MFPADs given simple assumptions about the bound states involved in the PI process and about the scattering process [30]. General characteristics of the CDAD are derived which indicate the relationship to the symmetry of the initial ionized orbital. The photoionization dynamics also play a role for some complementary features of the CDAD parameter. This analysis of the combined experimental and theoretical results, supported by features of the electron-ion kinetic energy correlation, enables us to discuss the symmetry assignment for the $\mathrm{B}$ state of $\mathrm{NO}^{+}$populated in the 23-25 eV photon energy region. Comparing further the predictions of the simple model with the experimental and MCSCI CDAD results for K-shell PI of diatomic molecules suggests that for inner-shell ionization the dichroism arises purely as a final state scattering effect, similar to the conclusion from the study of PECD for K-shell ionization of chiral molecules. 


\section{The vector correlation method}

The $\left(\mathbf{V}_{\mathbf{A}}, \mathbf{V}_{\mathbf{e}}, \mathbf{k}\right)$ vector correlation method consists of measuring for each DPI event the three components of the $\mathbf{V}_{\mathbf{A}}$ ionic fragment and $\mathbf{V}_{\mathbf{e}}$ photoelectron nascent velocity vectors, deduced from the arrival time and position of both particles in a double velocity spectrometer described in detail previously [31]. The data reported in this paper were recorded at the circularly polarized light SU5 undulator-based VUV beamline [32] at Super-ACO, operated in the two-bunch mode (period 120ns, pulse time width 800 ps). Using the Stokes parameters $\left(s_{0}, s_{1}, s_{2}, s_{3}\right)$ the circular polarization rate $s_{3} / s_{0}$ of the light was higher than 0.95 in the considered energy range (18-25 eV), the remaining part being mostly unpolarized [33]. Photoionization occurs at the intersection of the supersonic molecular beam SAPHIRS [34] and the light beam. Ions and electrons are extracted from the interaction region by a dc electric field $\mathbf{E}$ whose magnitude ensures a $4 \pi$ collection of both particles (here between $10 \mathrm{~V} / \mathrm{cm}$ and $50 \mathrm{~V} / \mathrm{cm}$ ), and guided to the two position sensitive detectors through an intermediate region where focusing lens sets were set to control the charged particle trajectories [31].

The coincident events for each studied DPI process were selected using the electron-ion kinetic energy correlation diagram as described in the earlier related papers $[35,36,40,41]$, and illustrated in sect. 6 for PI of $\mathrm{NO}$ at the 23.65 and $25 \mathrm{eV}$ photon excitation energies.

The three angle fit of the $I\left(\chi, \theta_{e}, \phi_{e}\right)$ angular distribution derived from the spatial analysis of the $\left(\mathbf{V}_{\mathbf{A}}, \mathbf{V}_{\mathbf{e}}, \mathbf{k}\right)$ vector correlation for the selected events, performed according to the general expression given in Eq.1, provides the five $F_{L N}\left(\theta_{e}\right)$ functions. In the present discussion we will use two representations of the circular dichroism results: (i) the CDAD parameter as defined in eq.2 and (ii) the cuts of the $I_{+1 \chi}=90\left(\theta_{e}, \phi_{e}\right)$ MFPAD for a molecule oriented

Deleted: 34
Deleted: 35
Deleted: 39
Deleted: 40




\section{Theory}

Theoretically, we will consider molecular frame photoelectron angular distributions (MFPADs) and in particular circular dichroism in molecular frame photoionization from two perspectives. First we will compute the MFPADs using ab initio methods, specifically using the multichannel Schwinger configuration interaction (MCSCI) method [37,38]. In smaller molecular systems, the MCSCI results can provide nearly quantitative agreement between the experiment and theory. This level of agreement has allowed for the study of dissociative lifetimes [39,40] and ion dynamics [41] through a comparison of measured and computed MFPADs. The second theoretical approach for studying MFPADs is through a simple model of the ionization process which allows for the description of the MFPAD using a few parameters with physical significance, including the partial-wave composition of the initial state, and scattering phase shifts estimated from quantum defects which correspond to the zero-energy scattering phase shifts for the various scattering partial-waves [30]. In the present paper we will focus in particular on CDADs obtained using these two approaches.

The MCSCI method has been developed over a number of years and can provide an accurate representation of scattering processes for linear molecular systems. This approach has been described in detail elsewhere [37,38] and here we will only discuss the general features of the method. When applied to the photoionization of a molecule with $N$ electrons, the MCSCI scattering wave function $\Psi_{k l m}^{(-)}(\vec{X}, \vec{r})$ is written in a close-coupling expansion where each term in the expansion is a product of a target ion state of $N-1$ electrons, $\Phi_{i}(\vec{X})$, and a one-electron continuum function $\psi_{i, k l m}^{(-)}(\vec{r})$ :

Deleted: 36

Deleted: 37

\begin{tabular}{|l|} 
Deleted: 38 \\
\hline Deleted: 39 \\
Deleted: 40
\end{tabular}

Deleted: describe

\begin{abstract}
perpendicular to the propagation axis of circularly polarized light (helicity +1$)($ see Eq.3) in
\end{abstract} Eq.3 


$$
\Psi_{k l m}^{(-)}(\vec{X}, \vec{r})=\sum_{i} \hat{A}\left(\Phi_{i}(\vec{X}) \psi_{i, k l m}^{(-)}(\vec{r})\right), \quad \text { Eq.4 }
$$

where $\vec{X}$ represents the coordinates of the $N-1$ electrons, $\vec{r}$ is the coordinates of the photoelectron, and $\hat{A}$ is the antisymmetrization operator. The index $k$ on the scattering wave functions represents the asymptotic magnitude of the momentum, the indices $l$ and $m$ indicate that the dependence of homogeneous solution of the scattering equations on the photoelectron emission direction $\hat{k}$ has the form $Y_{l m}(\hat{k})$, and the superscript $(-)$ indicates that the wave function has the appropriate boundary conditions for an ejected photoelectron. The ion states are each written as configuration-interaction expansions with a modest number of configurations, typically including 1000 to 10000 configuration state functions. The close coupling expansion is also restricted to a small number of important ion states. In the results presented here for the photoionization of NO, this expansion included 17 low-lying ion states [38,42]. In the calculations on $\mathrm{N}_{2} \mathrm{O}$, a more limited expansion was used with only five valence ion states [40].

$$
I_{l m \mu}=\left\langle\Psi_{0}\left|d_{\mu}\right| \Psi_{k l m}^{(-)}(\vec{X}, \vec{r})\right\rangle, \quad \text { Eq.5 }
$$

where $d_{\mu}$ is the dipole operator with angular momentum about the $z$ axis of $\hbar \mu$ are computed using the Schwinger variational method where all required integrals are numerically computed using a single-center expansion method [43]. The initial $N$ electron state $\Psi_{0}$ is also described by a CI wave function. In all applications to date, the initial and final target states were written using the same set of one-electron functions. This one-electron basis set is usually obtained from a complete-active-space self-consistent field (CASSCF) type calculation. The computed $I_{l m \mu}$ matrix elements can then be combined to compute the desired MFPAD or CDAD. 
In the simplest case for a linear molecule, the computed MFPAD is assumed to be the same as the measured recoil-frame photoelectron angular distribution (RFPAD). This is known as the axial-recoil approximation [44] $]_{y}$ However, when the lifetime of the dissociative state is a significant fraction of the rotational period of the molecule, the axial-recoil approximation is no longer valid and the effects of the rotation must be included in the calculation to allow for a direct comparison with experimental data [39,40]. In the case of the photoionization of NO considered here, one source of the discrepancy between theory and experiment may be long lifetimes of the ion states. Thus we have considered different dissociative lifetimes using a rotational constant of $1.67195 \mathrm{~cm}^{-1}$ [45] and rotational temperature of $35 \mathrm{~K}$ estimated for the molecular beam expansion.

In addition to the accurate MCSCI calculations, we will also consider a simple model (SM) [30] for the dipole transition matrix elements $I_{\operatorname{lm} \mu}$ in which a limited number of parameters are used. In this model, the matrix are assumed to have the form $I_{l m \mu}^{\mathrm{SM}}=i^{l} \exp \left\{-i\left[\eta_{l}(k)+\pi \tau_{l m}\right]\right\} \sum_{l^{\prime} m^{\prime}} C_{l^{\prime} m^{\prime}}\left\langle Y_{l^{\prime} m^{\prime}}\left|Y_{1 \mu}\right| Y_{l m}\right\rangle, \quad$ Eq.6 where $\eta_{l}(k)$ is the Coulomb scattering phase shift which depends on the asymptotic photoelectron momentum $k, \pi \tau_{l m}$ is the scattering phase, and $C_{l^{\prime} m^{\prime}}$ is related to the partialwave content of the initial orbital. The values of the scattering phases are assumed to be energy independent. The $\tau_{l m}$ are given by their low energy values which are known to smoothly connect to the quantum defects of the corresponding Rydberg states with angular momentum quantum numbers $l$ and $m$ that lie below the ionization threshold [46]. Thus we chose values of $\tau_{l m}$ that are typical of the quantum defects for small linear molecular systems. The derivation of equation Eq.6 also neglects any coupling between the continuum partial waves and assumes that the radial integrals involving different partial waves of the continuum functions and the same partial wave of the bound orbital all have the same value given by $C_{l^{\prime} m^{\prime}}$. The reference model assumes a set of scattering phase shifts with $\tau_{00}=1.0$, 
$\tau_{10}=\tau_{11}=0.7, \tau_{20}=0.2$, and the value of all other phase shifts are taken to be $\tau_{l m}=0$. We further note that the only energy dependence in the SM MFPADs comes from the energy dependence of the Coulomb phases shifts. Thus the present SM does not include resonant effects such as shape resonances and autoionization [25]. Such effects could be included in a SM formalism by including appropriate energy dependence in the magnitude and phases of the dipole matrix elements. Finally, we note that the SM does not include any effects from coupling between asymptotic channels that lead to different ion states. With this assumption, $\underline{\text { the initial state orbital correspond to the Dyson orbital, which implicitly includes all initial }}$ $\underline{\text { state and final ion state electron-electron correlation effects [47]. Thus the SM model can be }}$ applied to states with significant configuration interaction mixing in the target wave functions as long as interchannel coupling effects are small.4. Circular dichroism for photoionization Deleted: ף II of a sp $\sigma$ valence shell orbital

In Figure 2(a), we report the CDAD results for $\mathrm{PI}$ of $\mathrm{N}_{2} \mathrm{O}$ into the $\mathrm{N}_{2} \mathrm{O}^{+} C^{2} \Sigma^{+}(6 \sigma)^{-1}$ state: $\mathrm{N}_{2} \mathrm{O}\left(X^{1} \Sigma^{+}\right)+h v \rightarrow \mathrm{N}_{2} \mathrm{O}^{+}\left(C^{2} \Sigma^{+}(0,0,0)\right)+\mathrm{e} \rightarrow \mathrm{NO}^{+}\left(X^{1} \Sigma^{+}, \mathrm{v}\right)+\mathrm{N}\left({ }^{2} \mathrm{P}\right)+\mathrm{e}$ for a photon excitation energy $h v \approx 21 \mathrm{eV}$, corresponding to a photoelectron energy $\mathrm{E}_{\mathrm{e}} \approx 1$ $\mathrm{eV}$, in terms of the CDAD parameter and the cut of the $I_{+1 \chi=90}\left(\theta_{e}, \phi_{e}\right)$ MFPAD in the plane perpendicular to the light propagation axis $\left(\phi_{e}=90^{\circ}\right.$ and $\left.\phi_{e}=270^{\circ}\right)$ as defined in section 2 . This reaction was identified in our previous investigation of this system using linearly polarized light [40]. We note that for all the data presented in this paper, involving the $\mathrm{N}_{2} \mathrm{O}$ and the NO molecules, $\theta_{e}$ is the electron polar emission angle relative to the molecular axis oriented such that $\theta_{e}=0^{\circ}$ corresponds to an electron emission in the direction of the $\mathrm{N}$ end of the molecule as shown in Figure 2.

The measured circular dichroism for reaction Eq.7 displays a single positive oscillation, which reaches a maximum value of about 0.2 . The computed CDAD is also dominated by a single smooth positive oscillation, reaching significantly larger values with a maximum of 
about 1: however, taking into account the existence of a predissociation time of $2 \mathrm{ps}$ for the $C$

${ }^{2} \Sigma^{+}$ionic state and the subsequent rotation of the molecule prior to dissociation [40], the computed CDAD is decreased and matches very well the experimental result. The agreement is also very good at the level of the MFPADs when the effect of rotation is included in the calculation, illustrating the strong left-right emission anisotropy with a clear propensity for electron emission on the left side $\left(\phi_{e}=90^{\circ}\right)$, for a positive photon helicity.

The comparison of the circular dichroism for the reaction in Eq.7 with the one obtained previously for PI of the NO molecule into the $\mathrm{NO}^{+}\left(c^{3} \Pi\right)$ state according to :

$$
\mathrm{NO}\left(X^{2} \Pi\right)+h v \rightarrow \mathrm{NO}^{+}\left(c^{3} \Pi\right)+\mathrm{e} \rightarrow \mathrm{N}^{+}\left({ }^{3} \mathrm{P}\right)+\mathrm{O}\left({ }^{3} \mathrm{P}\right)+\mathrm{e} \quad \text { Eq. } 8
$$

for comparable photoelectron energies, here $h v \approx 23.65 \mathrm{eV}$ and $\mathrm{E}_{\mathrm{e}} \approx 1.8 \mathrm{eV}$ [35], also displayed in Figure 2 (b) exhibits a similar behavior: the measured CDAD reaches significant positive values, very well predicted by the calculation.

Figure 2 also displays the canonical Hartree Fock (HF) orbitals representing the $\mathrm{N}_{2} \mathrm{O}(6 \sigma)$ and the $\mathrm{NO}(4 \sigma)$ molecular orbitals (MOs), respectively. In $\mathrm{N}_{2} \mathrm{O}$, the $6 \sigma \mathrm{MO}$ in the region of the NO bond is very similar to the $4 \sigma \mathrm{MO}$ of NO. In both cases, the MO can be well characterized in a linear combination of atomic orbital (LCAO) description as being a N $2 s+$ O $2 p_{\mathrm{z}}$ orbital.

The qualitative features of the CDADs shown in Fig. 2 can be understood using the SM for the CDAD. In the SM, the initial state can be represented as a simple LCAO state with equal contributions of $s \sigma$ and $p \sigma$ atomic orbitals, i. e. with $C_{00}=+1.0$ and $C_{10}=+1.0$. The resulting CDAD is given in panel (a) of Fig. 3. We can see that the SM model CDAD is strongly positive for most angles. The fact that the CDAD is generally positive for the $C_{00}=+1.0$ and $C_{10}=+1.0$ initial orbital is a reflection of the relative phase of the $s \sigma$ and $p \sigma$ contributions to the initial orbital. If the phase of $p \sigma$ is changed so that the initial orbital is described as $C_{00}=+1.0$ and $C_{10}=-1.0$, the resulting CDAD is predominantly negative as 
seen with the dashed line in panel (a) of Fig. 3. The generally positive CDAD in the experimental data seen in Fig. 2 can then be attributed to the fact that the initial MOs as also shown in Fig. 2 have the valence part of the $\mathrm{N} 2 s$ with the same phase as the upper lobe of the O $2 p_{z}$ orbital. We point out that, although the shape of the MFPAD evolves significantly across the shape resonance region in the $25-40 \mathrm{eV}$ range, the CDAD remains dominantly positive as shown by the MCSCI calculations reported in [6], as well as the experimental result for hv $\approx 40 \mathrm{eV}[5]$.

\section{Circular dichroism for photoionization of a $p \pi$ valence shell orbital}

In Figure 4, we now compare the CDAD results for two PI reactions of $\mathrm{O}_{2}$ and $\mathrm{N}_{2} \mathrm{O}$ involving an ionized orbital of $\pi$ symmetry, for a photon excitation energy $h v \approx 24.4 \mathrm{eV}$ [24] and $19.5 \mathrm{eV}$ [25], respectively:

$$
\begin{gathered}
\mathrm{O}_{2}\left(\mathrm{X}^{3} \Sigma_{g}^{-}\right)+\mathrm{h} v \rightarrow \mathrm{O}_{2}^{+}\left(3{ }^{2} \Pi_{u}\right)+\mathrm{e} \rightarrow \mathrm{O}^{+}\left({ }^{2} P\right)+\mathrm{O}\left({ }^{3} P\right)+\mathrm{e} \\
\mathrm{N}_{2} \mathrm{O}\left(X^{1} \Sigma^{+}\right)+h v \rightarrow \mathrm{N}_{2} \mathrm{O}^{+}\left(B^{2} \Pi\right)+\mathrm{e} \rightarrow \mathrm{NO}^{+}\left(X^{1} \Sigma^{+}, \mathrm{v}\right)+\mathrm{N}\left({ }^{2} \mathrm{D}\right)+\mathrm{e}
\end{gathered}
$$

corresponding to photoelectron energies in the range of $\mathrm{E}_{\mathrm{e}} \approx 0.5-1.5 \mathrm{eV}$. For PI of $\mathrm{O}_{2}(\mathrm{Eq} .9)$ the CDAD parameter displays a clear oscillatory structure about 0 , with maxima and minima of magnitude of about 0.3 , corresponding to a very different $\theta_{e}$ dependence when compared to the one observed for ionization of the $s p \sigma$ orbitals shown in Figure 2. This structure is very well described by the MCSCI calculation, although the computed extrema values are larger. Indeed for the $\mathrm{O}_{2}$ homonuclear molecule, the intrinsic inversion symmetry of the CDAD parameter with respect to $\theta_{e}=90^{\circ}$, as well as that of the $F_{11}$ function, imposes at least a node for $\theta_{e}=90^{\circ}$, therefore an oscillatory structure. In such a case, the CDAD effect, when averaged over $\theta_{e}$, goes to zero.

For PI of $\mathrm{N}_{2} \mathrm{O}$ into the $\mathrm{N}_{2} \mathrm{O}^{+}\left(B^{2} \Pi\right)$ ionic state (Eq,10), also explored at a few other energies in the $18.5-21 \mathrm{eV}$ photon energy range, the measured CDAD parameter oscillates
Deleted: 2

Deleted: 1

Deleted: 1
Deleted: 1

Deleted: 6 
smoothly, and reaches significantly lower values. For this reaction we have shown previously that the molecule must bend prior to dissociation, corresponding to a mean recoil angle of $\theta_{R}$ $=30 \mathrm{deg}$. [41] and causing a significant attenuation of the emission anisotropies. The MCSCI calculations including the effect of bending prior to dissociation lead to a fair agreement with the experimental result, for the CDAD and the MFPADs.

The comparison between the computed circular dichroism effects for both reactions exhibit strong similarities: the oscillatory structure are quite comparable, although the inversion symmetry property is not strictly satisfied for PI into the $\mathrm{N}_{2} \mathrm{O}^{+}\left(B{ }^{2} \Pi\right)$.

The canonical Hartree-Fock orbitals that correspond to the initial orbitals from which the photoelectron is ejected in ionization leading to the $\mathrm{O}_{2}^{+}\left(3{ }^{2} \Pi_{u}\right)$ and $\mathrm{N}_{2} \mathrm{O}^{+}\left(B{ }^{2} \Pi\right)$ states are shown in Fig. 4. Both of these orbitals can be described as bonding $\pi$ orbitals that in the LCAO description would be the in-phase linear combination of $p \pi$ orbitals centered on each atom. In the SM for these systems we would thus represent the initial state by a simple $p \pi$ initial orbital. The predicted CDAD in the reference SM is given as the simple dashed line in panel (b) of Fig. 3. Although the SM CDAD does not resemble either the measured or computed MCSCI CDADs, we note that it satisfies the inversion symmetry with respect to $\theta_{e}$ $=90^{\circ}$ required for an homonuclear molecule, which is in fact characteristic of the initial orbital symmetry in the SM description, valid here both for $\mathrm{O}_{2}$ and $\mathrm{N}_{2} \mathrm{O} p \pi$ MOs. In general the SM will always predict a CDAD with this odd symmetry when the initial orbital has definite parity. Furthermore, we have previously noted [24] that the CDAD function is particularly sensitive to the relative phases of the photoelectron scattered waves. In panel (b) of Fig. 3, we give the CDAD for the $p \pi$ initial state when the assumed scattering phase shifts of the different symmetries of the $d$ waves are varied. A choice of $d$ wave phase shifts of $\tau_{20}=0.0, \tau_{21}=0.2$, and $\tau_{22}=-0.2$ gives SM CDAD results that are very similar to the measured and MCSCI CDADs for the two examples of ionization from $p \pi$ initial orbitals considered here. This modified SM result is very similar to that previously discussed [24]
Deleted: 40

Deleted: 3

Deleted: 2

Deleted: 2

Deleted: quantum defects

Deleted: quantum defects 
with respect to the ionization leading to the $\mathrm{O}_{2}{ }^{+}\left(3{ }^{2} \Pi_{u}\right)$ state. It also preserves the inversion symmetry property of the CDAD, which tends to zero when averaged over $\theta_{e}$. We now associate this characteristic to the (quasi) definite symmetry of the ionized orbital.

\section{Photoionization of $\mathrm{NO}$ into the $\mathrm{NO}^{+}(\mathrm{B})$ state}

The symmetry of the second dissociative ionic state populated by PI of the NO molecule in the $22.5-24 \mathrm{eV}$ region labeled as $\mathrm{NO}^{+}(\mathrm{B})$, and vibrationally resolved in photoelectron spectroscopy (PES) [26], has not been definitely assigned [28], to our knowledge. Two assignments have been proposed in literature, namely the $\Sigma$ symmetry $\left(\mathrm{B}^{\prime}{ }^{1} \Sigma^{+}\right)[26]$ and the $\Pi$ symmetry (B ${ }^{1} \Pi$ ) [27] corresponding to ionization of the $1 \pi$ orbital and the $4 \sigma \mathrm{MO}$ discussed for $\mathrm{Eq}, 8$, respectively. In the calculations by Partridge et al [29], the vibrational distribution extending in the 22.6-23.6 energy range in the PES spectra is attributed to the (B $\left.{ }^{1} \Pi\right)$ state. In this section we report and discuss the CDAD for this process for the $h v \approx 23.65 \mathrm{eV}$ photon energy, with reference to the CDAD properties that we have characterized for ionized MOs of different symmetries in sections 4 and 5 . At the $h v \approx 23.65 \mathrm{eV}$ photon energy, PI into the B state of $\mathrm{NO}^{+}$involves precisely the 22.6-23.6 binding energy range, corresponding to photoelectrons in the $0-1 \mathrm{eV}$ energy range, as shown on the electron-ion kinetic energy correlation diagram (KECD) derived from the vector correlation analysis, displayed in Figure 5(a). The KECD shows that the B state predominately dissociates into the $\mathrm{L}_{1}$ ground state limit channel as reported earlier [35, 48$]$ :

$$
\mathrm{NO}\left(X^{2} \Pi\right)+h v \rightarrow \mathrm{NO}^{+}(\mathrm{B})+\mathrm{e} \rightarrow \mathrm{N}^{+}\left({ }^{3} \mathrm{P}\right)+\mathrm{O}\left({ }^{3} \mathrm{P}\right)+\mathrm{e} \quad \mathrm{Eq}, 11
$$

The photoelectron energy resolution does not enable us to resolve the vibrational distribution. The KECD obtained for a photon energy $h v \approx 25 \mathrm{eV}$ is reported in Fig 5 (b) to supply complementary information for PI in this region. Now the 22.6-23.6 binding energy range attributed to the B state discussed here corresponds to photoelectrons of 1.4-2.4 eV energy.

Deleted: 4

Deleted: 34

Deleted: 47

Deleted: 8 
The KECD also shows that the diagonal structure along the $\mathrm{L}_{1}$ dissociation channel extends until the zero electron energy limit, characterizing the population of a strongly repulsive ionic state in the FC region. This peak is consistent with the underlying continuous photoelectron energy distribution in the PES spectra [26]. Some features of the MFPADs for the two 22.6$23.6 \mathrm{eV}$ and 23.6-25 eV components suggest the contribution of two different electronic states. One sees also that a third electronic state lying at about $24.8 \mathrm{eV}$ in the FC region is populated, which dissociates into the $\mathrm{L}_{2} / \mathrm{L}_{3}\left(\mathrm{~N}^{+}\left({ }^{1} \mathrm{D}\right)+\mathrm{O}\left({ }^{3} \mathrm{P}\right)\right.$ and/or $\left.\mathrm{N}^{+}\left({ }^{3} \mathrm{P}\right)+\mathrm{O}\left({ }^{1} \mathrm{D}\right)\right)$ close lying dissociation channels.

Figure 6 displays the $4 \sigma$ and $1 \pi$ canonical Hartree-Fock orbitals (a,b), the MCSCI computed CDADs for PI into the $\left(\mathrm{B}^{1} \Pi\right)$ and $\left(\mathrm{B}^{,}{ }^{1} \Sigma^{+}\right)$ionic states $(\mathrm{c}, \mathrm{d})$ for an electron energy about $0.5 \mathrm{eV}$, and the measured CDAD (e) at $23.65 \mathrm{eV}$. The MCSCI CDADs are consistent with the fingerprints reported for the $\operatorname{sp} \sigma$ and $p \pi$ orbitals in sect. 4 and 5 , corresponding to a strong positive circular dichroism for the $4 \sigma \mathrm{MO}$, and an oscillatory structure presenting a quasi-inversion symmetry for the $1 \pi$ MO. Besides their different CDADs, the $\left(\mathrm{B}^{1} \Pi\right)$ and $\left(\mathrm{B}^{\text {' }}\right.$ ${ }^{1} \Sigma^{+}$) states correspond to a parallel and a perpendicular transition with large asymmetry parameters, $\beta_{\mathrm{n}} \approx 1$ and $\beta_{\mathrm{n}} \approx-0.4$ in the MCSCI calculation, respectively. They are also characterized by $F_{22}$ functions of opposite signs: $F_{22}>0$ for the $\mathrm{NO}\left(X^{2} \Pi\right) \rightarrow \mathrm{NO}^{+}\left(\mathrm{B}^{1} \Pi\right)$ transition, and $F_{22}<0$ for the $\mathrm{NO}\left(X^{2} \Pi\right) \rightarrow \mathrm{NO}^{+}\left(\mathrm{B}^{,}{ }^{1} \Sigma^{+}\right)$transition.

The measured CDAD reported in Figure 6(e) shows two smooth positive oscillations with weak maxima about 0.1 , and cannot be assigned directly to ionization of the $4 \sigma$ or the $1 \pi$ orbital. This observation suggests that the PI process in the 22.6-23.6 eV energy region corresponds predominately to PI into the $\left(\mathrm{B}^{1} \Pi\right)$ state, assuming a predissociation time at least comparable to the rotation period, that significantly reduces the magnitude of the circular dichroism, as illustrated in Figure 6(c). The MCSCI calculations including the effect of rotation lead to a consistent agreement with the experimental result, for the CDAD and the MFPADs (Fig.6(e), (f)). This interpretation is supported by the positive measured photoion 
asymmetry parameter $\beta_{\mathrm{N}^{+}} \approx 0.35 \pm 0.1$ and the positive sign of the measured $F_{22}$ function. The slight negative $\mathrm{CDAD}$ value about $\theta_{e} \approx 90^{\circ}$, as well as other quantitative features of the

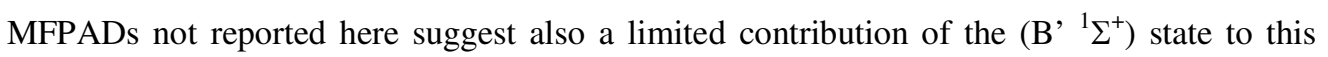
region. The complete MFPAD results will be reported later, as well as those for the two components of the elongated structure observed at $\mathrm{h} v \approx 25 \mathrm{eV}$ : they support the assignment of the 23.6-25 eV binding energy region to the dominant contribution of the $\left(\mathrm{B}^{1}{ }^{1} \Sigma^{+}\right)$state. This complex ionization continuum region deserves complementary studies at higher resolution, and a more detailed comparison with MCSCI calculations taking into account the several electron energies involved in ionization into repulsive ionic states, such as the $\left(B{ }^{1} \Pi\right)$ and $\left(B^{\prime}\right.$ $\left.{ }^{1} \Sigma^{+}\right)$states.

\section{Discussion and conclusion}

In this paper we have reported a detailed comparative study of experimental and theoretical results for molecular frame photoemission induced by VUV circularly polarized light for valence shell PI of selected simple molecules. The vector correlation method on the experimental side has been combined with the MCSCI $a b$ initio method and the predictions of a simple model for photoionization, on the theoretical side. This study enables us to emphasize some general qualitative features of circular dichroism in molecular frame photoemission, illustrating both the influence of the initial molecular orbital symmetry and the sensitivity of the final state scattering effect. Disentangling the role of the initial MO properties, we conclude that a strong positive (or negative) circular dichroism effect, that produces a significant left-right electron emission asymmetry in the plane perpendicular to the propagation axis of circularly polarized light, even after averaging over the polar angle $\theta_{e}$, requires that the initial MO has no definite symmetry. This is the case for example for an initial $s p \sigma$ orbital as illustrated in PI of $\mathrm{NO}$ and $\mathrm{N}_{2} \mathrm{O}$ into the $\mathrm{NO}^{+}\left(c^{3} \Pi\right.$ and $\left.B{ }^{1} \Pi\right)$ and $\mathrm{N}_{2} \mathrm{O}^{+}$ $\left(C^{2} \Sigma^{+}(0,0,0)\right)$ ionic states, respectively. On the other hand, when the ionized orbital 
possesses a (quasi) defined parity like the $p \pi$ orbital involved in PI of $\mathrm{O}_{2}$ and $\mathrm{N}_{2} \mathrm{O}$ into the $\mathrm{O}_{2}^{+}\left(3{ }^{2} \Pi_{u}\right)$ and $\mathrm{N}_{2} \mathrm{O}^{+}\left(B^{2} \Pi\right)$ states, the CDAD must display an oscillatory structure with an odd symmetry with respect to $\theta_{e}=90^{\circ}$, whose integral value is zero, or near zero. This is clearly the case, although the double oscillation observed differs from single oscillation obtained at the simplest level of the SM. However, a slight modification of the relative phases of the photoelectron scattered waves, here the $d \lambda$ partial waves, enables a very good description of the CDAD, pointing to the sensitivity of the CDAD to the scattering dynamics.

Based on this analysis, the results obtained in the study of PI of NO in the $22-25 \mathrm{eV}$ energy region favor the assignment of the ionic state corresponding to $22.6-23.6 \mathrm{eV}$ binding energies predominately to the $\mathrm{NO}^{+}\left(B^{1} \Pi\right)[27,29]$. However, this specific case remains rather complex since the ionic states involved must have a significant predissociation time, and a partial overlap, and it would require further investigation.

We now consider briefly the case of an $s \sigma$ ionized orbital, that we did not discuss earlier in the paper. As shown in Figure $3(\mathrm{c})$, the SM predicts a CDAD equal to zero, unless a phase shift is introduced between the two $p \sigma$ and $p \pi$ partial waves in the final scattering state, leading to a CDAD that is very similar to the CDAD for the initial $p \pi$ orbital without modified phase shifts (Fig $3(\mathrm{~b}))$. Both of these CDADs have the odd symmetry characteristic of the ionization from an orbital with definite symmetry, but in the case of the $s \sigma$ ionization the change of the phase shifts between the $p \sigma$ and $p \pi$ waves cannot induce the double oscillation structure seen in the $p \pi$ orbital CDAD with modified phase shifts. We note however that for K-shell ionization, a significant CDAD displaying a strong oscillatory structure has been observed for diatomic molecules e.g. $\mathrm{N}_{2}$ and $\mathrm{CO}$ (C 1s orbital) [4] which, for the dominant features, changes sign when reflected through $\theta_{e} \approx 90^{\circ}$; this is also the case to some extent only for $\mathrm{N} 1 \mathrm{~s}$ and $\mathrm{O} 1 \mathrm{~s}$ ionization of NO [22,23]. The number of oscillations found implies the contribution of higher $l$ partial waves, showing the importance of the final state scattering effects which are outside the scope of the SM. This discussion suggests that

Deleted: quantum defects Deleted: 2

Deleted: quantum defects

Deleted: quantum defects 
for $1 \mathrm{~s} \mathrm{K-shell} \mathrm{ionization} \mathrm{the} \mathrm{CDAD} \mathrm{arises} \mathrm{purely} \mathrm{as} \mathrm{a} \mathrm{final} \mathrm{state} \mathrm{scattering} \mathrm{effect,} \mathrm{quite} \mathrm{similar}$ to the conclusion from the study of PECD for K-shell ionization of chiral molecules [18, $19,49]$.

Further investigations will be interesting to pursue in order to explore the general validity of the qualitative features of the molecular frame CDAD for achiral molecules reported here, and their power to characterize the relative importance of the properties of the initial MOs involved in photoionization of molecules and of the scattering dynamics on the other side. Site selected localized K-shell ionization of small polyatomic molecules will constitute one example [22]. Furthermore, although the origin of circular dichroism is different, it would be interesting to explore the circular dichroism for simple chiral systems in the recoil frame of the fragmenting molecule [50], that will differ from the molecular frame for non-linear molecules in general. The relative importance of the PECD induced by the intrinsic chirality of the system, and that of the CDAD due to the spatial geometry discussed here, has been investigated recently for core level PI of chiral molecules adsorbed on surfaces [51,52]. Such investigations would be very interesting to perform for valence-shell PI of simple chiral molecules in the gas phase.

\section{Acknowledgments}

We are very grateful to L. Nahon, beamline scientist for SU5 at Super-ACO (future DESIRS beamline at the synchrotron center SOLEIL), for fruitful discussions on PECD. We acknowledge $\mathrm{H}$. Lefebvre-Brion for a nice discussion about the spectroscopy of $\mathrm{NO}^{+}$. We thank B. Pilette at LURE for technical assistance, as well the machine department staff for operating Super-ACO (LURE). The support of the National Science Foundation (USA) through Grant. No. INT-0089831 and the support of the Centre National de la Recherche Scientifique (France) are gratefully acknowledged. RRL acknowledge the support of the Robert A. Welch Foundation (Houston, Texas) under grant A-1020. 


\section{References}

[1] K.L. Reid, Annu.Rev.Phys.Chem. 54, 397 (2003); R.E. Continetti, Annu. Rev. Phys. Chem. 52, 165 (2001); J. Ullrich, R. Moshammer, A. Dorn, R. Dörner, L. Ph. Schmidt and H. Schmidt-Böcking, Rep. Prog. Phys. 66, 1463 (2003); D. Dowek, in J. Ullrich and V.P. Shevelko (Eds.): Many-Particle Quantum Dynamics in Atomic and Molecular Fragmentation (Springer, Berlin-Heidelberg 2003), and ref. therein.

[2] K.L. Reid, D.J. Leahy, and R.N. Zare, Phys. Rev. Lett. 68, 3527 (1992).

[3] S. Motoki, J. Adachi, K. Ito, K. Ishii, K. Soejima, A. Yagishita, S.K. Semenov, and N.A. Cherepkov, Phys. Rev. Lett. 88, 063003 (2002).

[4] T. Jahnke, Th. Weber, A.L. Landers, A. Knapp, S. Schössler, J. Nickles, S. Kammer, O. Jagutzki, L. Schmidt, A. Czasch, T. Osipov, E. Arenholz, A.T. Young, R. Diez Muino, D. Rolles, F.J. Garcia de Abajo, C.S. Fadley, M.A. Van Hove, S.K. Semenov, N.A. Cherepkov, J. Rösch, M.H. Prior, H. Schmidt-Böcking, C.L. Cocke, and R. Dörner, Phys. Rev. Lett. 88, 073002 (2002).

[5] O. Gessner, Y. Hikosaka, B. Zimmermann, A. Hempelmann, R.R. Lucchese, J.H.D. Eland, P.M. Guyon, and U. Becker, Phys. Rev. Lett. 88, 193002 (2002).

[6] M. Lebech, J.C. Houver, A. Lafosse, C. Alcaraz, L. Nahon, R.R. Lucchese and D. Dowek, J. Chem. Phys. 118, 9653 (2003).

[7] L.D. Barron, Molecular Light Scattering and Optical Activity, $2^{\text {nd }}$ ed. (Cambridge University Press, Cambridge, 2004)

[8] T. Muller, K.B. Wiberg, P.H. Vaccaro, J.R. Cheeseman, and M.J. Fritsch, J. Opt. Soc. Am. B 19, 125 (2002)

[9] e.g. S.B. Nielsen, T. Chakraborty, and S.V. Hoffmann, ChemPhysChem 6, 2619 (2005) and ref. therein.

[10] G. Schönhense, Physica Scripta. T31, 255 (1990) 
[11] B. Ritchie, Phys. Rev. A 13, 1411 (1976).

[12] N.A. Cherepkov, Chem. Phys. Lett. 87, 344 (1982); N.A. Cherepkov, V.V. Kuznetsov, Z. Phys. D 7, 271 (1987).

[13] I. Powis, J. Chem. Phys. 112, 301 (2000); I. Powis, J. Phys. Chem. A 104, 878 (2000).

[14] N. Böwering, T. Lischke, B. Schmidtke, N. Müller, T. Khalil, and U. Heinzmann, Phys. Rev. Lett. 86 (7), 1187 (2001).

[15] G.A. Garcia, L. Nahon, M. Lebech, J.C. Houver, D. Dowek, and I. Powis, J. Chem. Phys. 119, 8781 (2003).

[16] S. Stranges, S. Turchini, M. Alagia, G. Alberti, G. Contini, P. Decleva, G. Fronzoni, M. Stener, N. Zema, and T. Prosperi, J. Chem. Phys. 122, 244303 (2005).

[17] L. Nahon, G.Garcia, C.J. Harding, E. Mikajlo, and I. Powis, J. Chem. Phys. 125, 114309 (2006).

[18] U. Hergenhahn, E. E. Rennie, O. Kugeler, S. Marburger, T. Lischke, I. Powis, and G. A. Garcia, J. Chem. Phys. 120, 4553 (2004).

[19] M. Stener, G. Fronzoni, D. Di Tommaso, and P. Decleva, J. Chem. Phys. 120, 3284 (2004).

[20] R.L. Dubs, S.N. Dixit, and V. McKoy, Phys. Rev. Lett. 54, 1249 (1985).

[21] C. Westphal, J. Bansmann, M. Getzlaff, and G. Schönhense, Phys. Rev. Lett. 63, 151 (1989).

[22] W.B. Li, J.C. Houver, A. Haouas, F. Catoire, C. Elkharrat, R. Guillemin, L. Journel, R. Montuoro, R.R. Lucchese, M. Simon, and D. Dowek, J. Electron Spectrosc. Relat. Phenom. in press.

[23] W.B. Li, J.C. Houver, L. Journel, A. Haouas, R. Montuoro, R.R. Lucchese, M. Simon, and D. Dowek, Phys. Rev. A to be published (2007).

[24] D. Dowek, M. Lebech, J.C. Houver, R.R. Lucchese, J. Electron Spectrosc. Relat. Phenom. 141, 211 (2004). 
[25] M. Lebech, J.C. Houver, D. Dowek and R.R. Lucchese, Phys. Rev. Lett. 96, 073001 (2006).

[26] O. Edqvist, L. Asbrink, and E. Lindholm, Z. Naturforsch. Teil A 26, 1407 (1971).

[27] H. Lefebvvre-Brion, Chem. Phys. Lett. 9, 463 (1971); P.W. Thulstrup et al, J. Chem. Phys. 60, 3975 (1974).

[28] D.L. Albritton, A.L. Schmeltekopf, and R.N. Zare, J. Chem. Phys. 71, 3271 (1979) and ref. therein.

[29] H. Partridge, S. R. Langhoff, and C.W. Bauschlicher, Jr, J. Chem. Phys. 93, 7179 (1990).

[30] R. R. Lucchese, J. Electron Spectrosc. Relat. Phenom. 141, 201 (2004).

[31] M. Lebech, J.C. Houver and D. Dowek, Rev. Sci. Instrum. 73, 1866 (2002)

[32] L. Nahon, C. Alcaraz, J.-L. Marlats, B. Lagarde, F. Polack, R. Thissen, D. Lepère, and K. Ito, Rev. Sci. Instrum. 72, 1320 (2001).

[33] L. Nahon and C. Alcaraz, Applied Optics, 43, 1024 (2004).

[34] M. Richard-Viard, A. Delboulbé, and M. Vervloet, Chem. Phys. 209, 159 (1996).

[35] A. Lafosse, M. Lebech, J.C. Brenot, P.M. Guyon, O. Jakutzki, L. Spielberger, M. Vervloet, J.C. Houver and D. Dowek, Phys. Rev. Lett. 84, 5987 (2000).

[36] A. Lafosse, J.C. Brenot, A.V. Golovin, P.M. Guyon, K. Hoejrup, J.C. Houver, M. Deleted: 35 Lebech and D. Dowek, J. Chem. Phys. 114 (15), 6605 (2001)

[37] R. E. Stratmann and R. R. Lucchese, J. Chem. Phys. 102, 8493 (1995).

[38] R. E. Stratmann, R. W. Zurales, and R. R. Lucchese, J. Chem. Phys. 104, 8989 (1996).

[39] A. Lafosse, J. C. Brenot, P. M. Guyon, J. C. Houver, A. V. Golovin, M. Lebech, D. Dowek, P. Lin, R. R. Lucchese, J. Chem. Phys. 117, 8368 (2002).

[40] M. Lebech, J. C. Houver, R. R. Lucchese, and D. Dowek, J. Chem. Phys. 117, 9248

Deleted: 39 (2002).

[41] M. Lebech, J.C. Houver, and D. Dowek, and R. R. Lucchese, J. Chem. Phys. 120, 8226

Deleted: 40 (2004). 
[42] R. R. Lucchese, A. Lafosse, J. C. Brenot, P. M. Guyon, J. C. Houver, M. Lebech, G.

[45] K. P. Huber and G. Herzberg, Molecular Spectra and Molecular Structure. IV (Van

[46] C. Jungen, Molecular Applications of Quantum Defect Theory (Taylor and Francis, New York, 1996).

[47] G. M. Seabra, I. G. Kaplan, V. G. Zakrzewski, and J. V. Ortiz, J. Chem. Phys. 121, 4143 (2004),48] J.H.D. Eland and E.J. Duerr, Chem. Phys. 229, 1 (1998).

[49] M. Stener, D. Di Tommaso, G. Fronzoni, P. Decleva and I. Powis, J. Chem. Phys., 124 024326 (2006).

[50] D. Toffoli, R.R. Lucchese, M. Lebech, J.C. Houver, D. Dowek, J. Chem. Phys. 126, 054307 (2007).

[51] M. Polcik, F. Allegretti, D.I. Sayago, G. Nisbet, C.L.A. Lamont, and D.P. Woodruff, Phys. Rev. Lett. 92, 236103 (2004).

[52] J.W. Kim, M. Carbone, J.H. Dil, R. Flammini, M.P. Casaletto, K. Horn, and M.N. 


\section{Figure captions}

Figure 1. Sketch of the triplet $\left(\mathbf{k}_{\mathbf{e}}, \mathbf{n}, \mathbf{k}\right): \chi$ is the polar angle referring the orientation of the molecular axis $\mathbf{n}$ with respect to the light propagation axis $\mathbf{k}$, the polar and azimuthal angles $\left.\underline{\left(\theta_{\mathrm{e}}\right.}, \phi_{\mathrm{e}}\right)$ characterize the $\mathbf{k}_{\mathbf{e}}$ photoelectron momentum direction in the $(\mathrm{x}, \mathrm{y}, \mathrm{z})$ molecular frame defined by the molecular axis and the light propagation axis $\left(0 \leq \chi \leq \pi ; 0 \leq \theta_{\mathrm{e}} \leq \pi ; 0 \leq \phi_{\mathrm{e}} \leq\right.$ $2 \pi)$ Figure 2. (a) $\mathrm{N}_{2} \mathrm{O}(6 \sigma)$ canonical HF orbital, $\mathrm{CDAD}$ and $\left(\phi_{\underline{e}}=90^{\circ} / \phi_{e}=270^{\circ}\right)$ cut of the $\mathrm{I}\left(\chi, \theta_{e}, \phi_{e}\right)$ MFPAD for a $\mathrm{N}_{2} \mathrm{O}$ molecule oriented perpendicular $\left(\chi=90^{\circ}\right)$ to the propagation axis of LHC circularly polarized light $(h=1)$ as shown (N up) for ionization of the $(6 \sigma)$ orbital: experimental result at $h v=21 \mathrm{eV}$ (dots), MCSCI calculations for $\mathrm{E}_{\mathrm{e}}=0.8 \mathrm{eV}$ (solid line), MCSCI result for a predissociation time $\tau=2$ ps (dashed line); (b) $\mathrm{NO}(4 \sigma)$ canonical $\mathrm{HF}$ orbital, $\mathrm{CDAD}$ and $\left(\phi_{e}=90^{\circ} / \phi_{e}=270^{\circ}\right)$ cut of the $\mathrm{I}\left(\chi, \theta_{e}, \phi_{e}\right)$ MFPAD for a NO molecule oriented perpendicular $\left(\chi=90^{\circ}\right)$ to the propagation axis of circularly polarized light $(h=1)$ as shown ( $\mathrm{N}$ up) for ionization of the $(4 \sigma)$ orbital: experimental results at $h v=23.65$ $\mathrm{eV}$ (dots), MCSCI calculations for $\mathrm{E}_{\mathrm{e}}=1.9 \mathrm{eV}$ (solid line); (see text); for the MFPADs, theory and experiment are normalized such that the total photoionization cross sections for each PI process are identical.

Figure 3. Predicted CDADs from the SM.

(a) CDAD for an initial ( $s p) \sigma$ orbital with $C_{00}=1.0, C_{10}= \pm 1.0$ and all $C_{l^{\prime} m^{\prime}}=0$ using the reference phase shifts: $C_{10}=+1.0\left(\right.$ solid line), $C_{10}=-1.0$ ( dashed line).

(b) CDAD for an initial $p \pi$ orbital with $C_{11}=1.0$ and all $C_{l^{\prime} m^{\prime}}=0.0$ :

- - - - the reference model with $\tau_{20}=0.2, \tau_{21}=0.0$, and $\tau_{22}=0.0$;

$\overline{-\ldots-} \underline{\tau_{20}=0.0}, \underline{\tau_{21}=0.0, \text { and }} \underline{\tau_{22}=0.0 ;}$
$\overline{\tau_{20}=0.0}, \underline{\tau_{21}=0.0 \text {, and }} \underline{\tau_{22}=-0.2 ;}$
$\overline{\tau_{20}=0.0,}, \tau_{21}=0.2$, and $\tau_{22}=-0.2 ;$
Formatted

Deleted:

Deleted: 1

Deleted: 2

Deleted: quantum defects 
(c) CDAD for an initial $s \sigma$ orbital with $C_{00}=1.0$ and all $C_{l^{\prime} m^{\prime}}=0$. reference model with $\tau_{10}=\tau_{11}=0.7$ (dashed line), $\tau_{10}=0.7$ and $\tau_{11}=0.9$ (solid line).

Figure 4. (a) $\mathrm{O}_{2}\left(1 \pi_{\mathrm{u}}\right)$ canonical HF orbital, $\mathrm{CDAD}$ and $\left(\phi_{e}=90^{\circ} / \phi_{e}=270^{\circ}\right)$ cut of the $\mathrm{I}(\chi$, $\left.\theta_{e}, \phi_{e}\right)$ MFPAD for a $\mathrm{O}_{2}$ molecule oriented perpendicular $\left(\chi=90^{\circ}\right)$ to the propagation axis of circularly polarized light $(h=1)$ for ionization of the $\left(1 \pi_{\mathrm{u}}\right)$ orbital: experimental results at $h v$ $=24.4 \mathrm{eV}$ (dots), $\mathrm{MCSCI}$ calculations for $\mathrm{E}_{\mathrm{e}}=0.7 \mathrm{eV}$ (solid line); (b) $\mathrm{N}_{2} \mathrm{O}(1 \pi)$ canonical HF orbital, $\mathrm{CDAD}$ and $\left(\phi_{e}=90^{\circ} / \phi_{e}=270^{\circ}\right)$ cut of the $\mathrm{I}\left(\chi, \theta_{e}, \phi_{e}\right)$ MFPAD for a $\mathrm{N}_{2} \mathrm{O}$ molecule oriented perpendicular $\left(\chi=90^{\circ}\right)$ to the propagation axis of circularly polarized light $(h=1)$ as shown ( $\mathrm{N}$ up) for ionization of the $(1 \pi)$ orbital: experimental results $h v=19.5 \mathrm{eV}$ (dots), MCSCI calculations for $\mathrm{E}_{\mathrm{e}}=1.2 \mathrm{eV}$ (solid line), MCSCI result for a mean recoil angle $\theta_{R}=$ 30 deg. (dashed line); (see text); for the MFPADs, theory and experiment are normalized such that the total photoionization cross sections for each PI process are identical.

Figure 5. $\left(\mathrm{E}_{\mathrm{e}}, \mathrm{E}_{\mathrm{N}+}\right)$ KECDs of the $\left(\mathrm{N}^{+}, \mathrm{e}\right)$ coincident events for DPI of NO at a photon excitation energy $h v=23.65 \mathrm{eV}$ (a) and $h v=25 \mathrm{eV}$ (b); the extraction field of $35 \mathrm{~V} / \mathrm{cm}$ combined with $\Lambda_{\mathrm{e}}$ and $\Lambda_{\mathrm{A}+}$ standard focusing lens sets ensures a $4 \pi$ collection of electrons and ions with $\mathrm{E} \leq 3 \mathrm{eV} . \mathrm{L}_{1}, \mathrm{~L}_{2} / \mathrm{L}_{3}$ correspond to the lowest dissociation limits (see text). The intensity scale runs from blank (lowest int.) to black (highest int.) in linear scale; within each KECD selection, the scales are consistent and the contour lines are spaced by $10 \%$ of the maximum value.

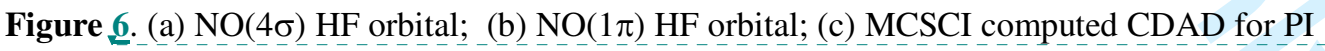
into the $\mathrm{NO}^{+}\left(\mathrm{B}^{1} \Pi\right)$ state for $\mathrm{E}_{\mathrm{e}} \approx 0.5 \mathrm{eV}$ and a predissociation time $\tau=0 \mathrm{ps}$ (solid line), $0.5 \mathrm{ps}$ (dotted line) and infinite (dashed line); (d) MCSCI computed CDAD for PI into the $\mathrm{NO}^{+}(\mathrm{B}$ ' 
${ }^{1} \Sigma^{+}$) state for $\mathrm{E}_{\mathrm{e}} \approx 0.5 \mathrm{eV}$ and a predissociation time $\tau=0$ ps (solid line), and infinite (dashed line); (e) measured CDAD at $h v=23.65 \mathrm{eV}$ (dots) for the selection of peak B in the KECD as shown in Fig. 5(a), compared with the MCSCI result for PI into the $\mathrm{NO}^{+}\left(\mathrm{B}^{1} \Pi\right)$ state $\left(\mathrm{E}_{\mathrm{e}} \approx 0.5\right.$ $\mathrm{eV}$ and $\tau$ infinite); (f) measured cut of the MFPAD (see caption of Fig. 2 and 4 ) at $h v=23.65$ Deleted: 4

$\mathrm{eV}$ for the selection of peak B (dots), compared with the MCSCI result for PI into the $\mathrm{NO}^{+}(\mathrm{B}$ $\left.{ }^{1} \Pi\right)$ state $(\mathrm{Ee} \approx 0.5 \mathrm{eV}$ and $\tau$ infinite) (solid line). 


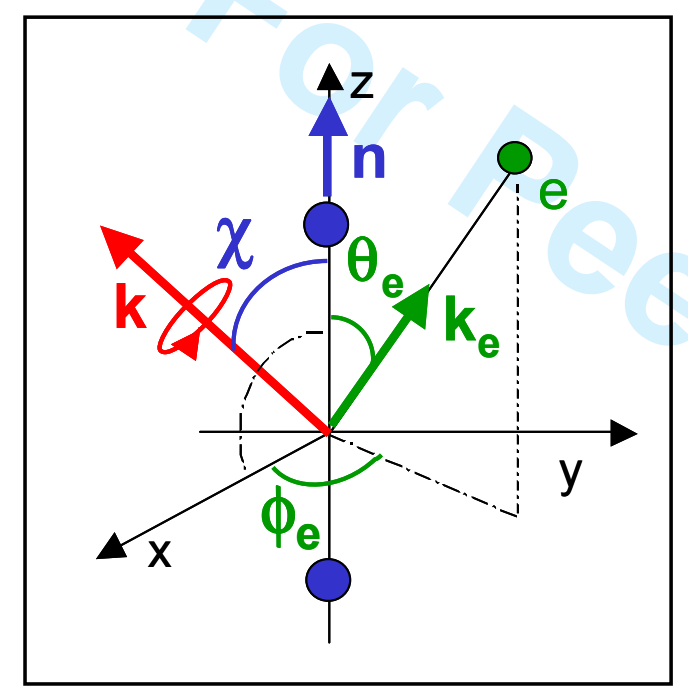

Figure 1 
(a)

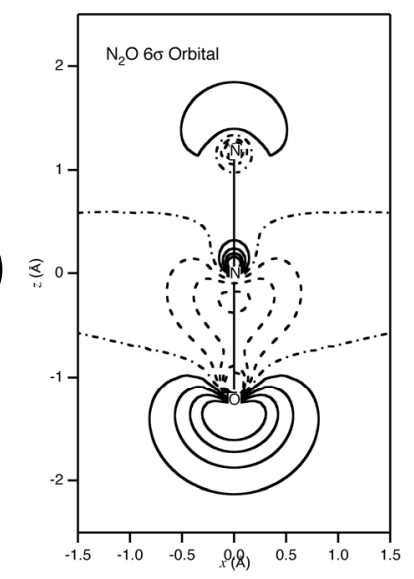

(b)

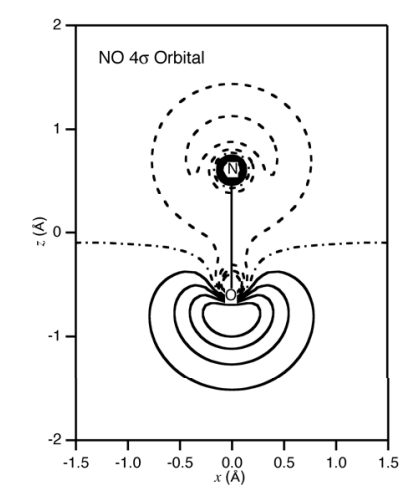

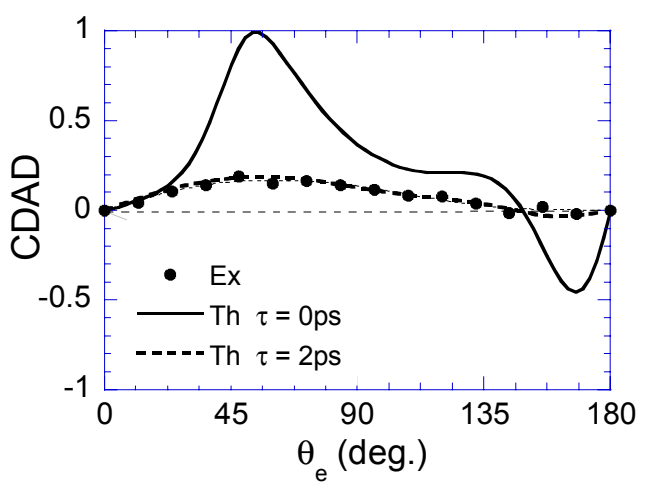
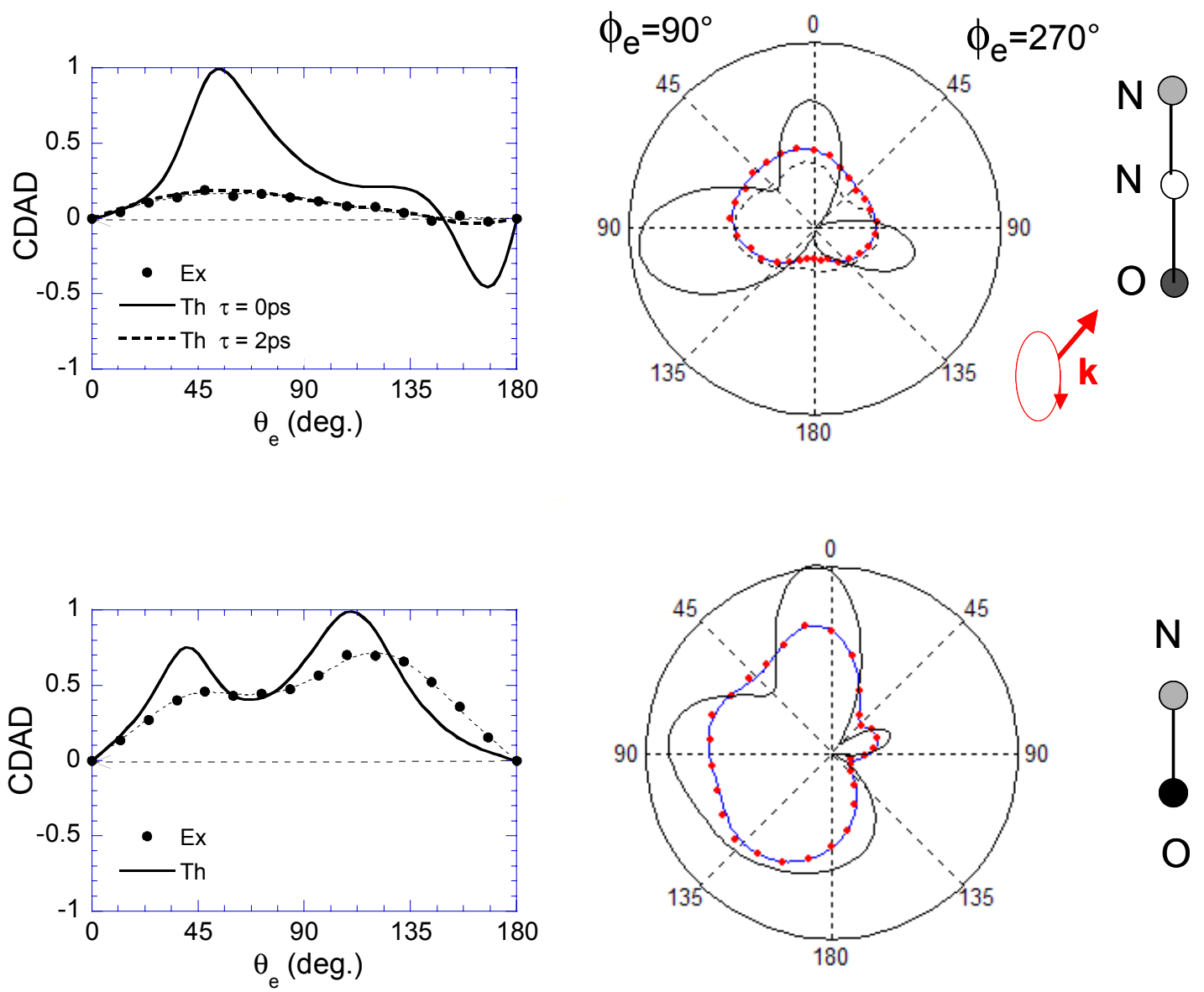

Figure 2 


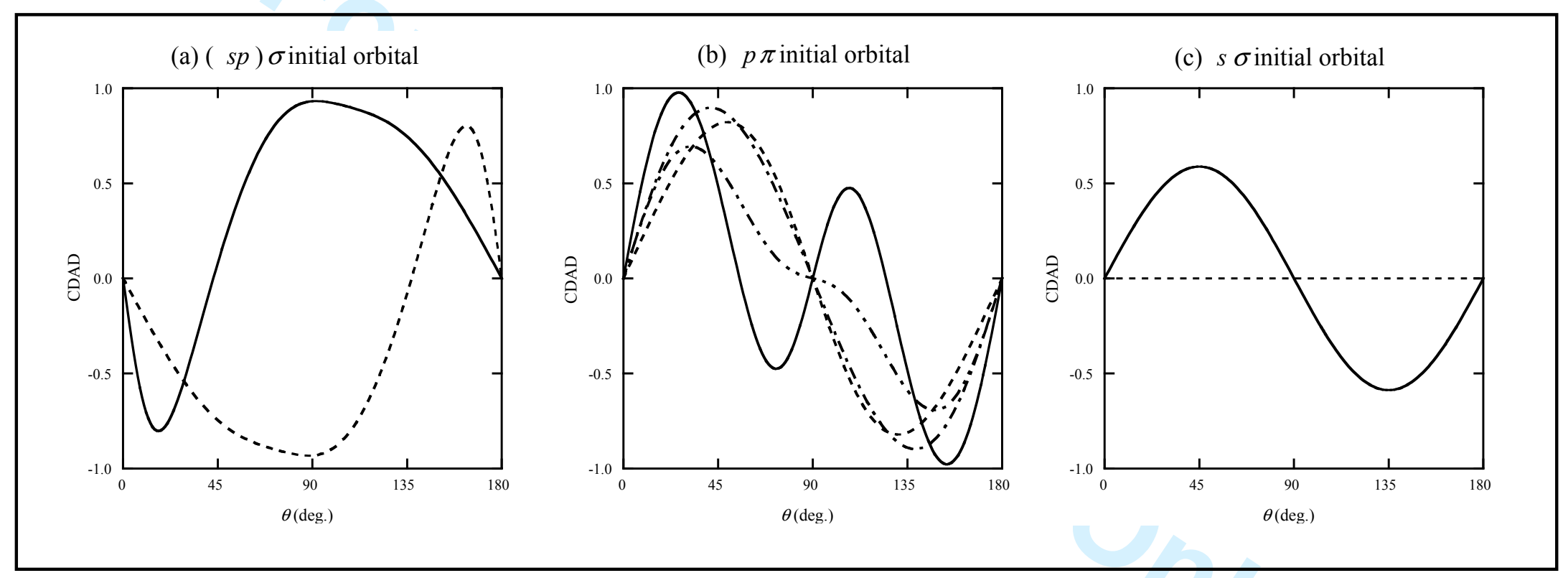

Figure 3 
(a)
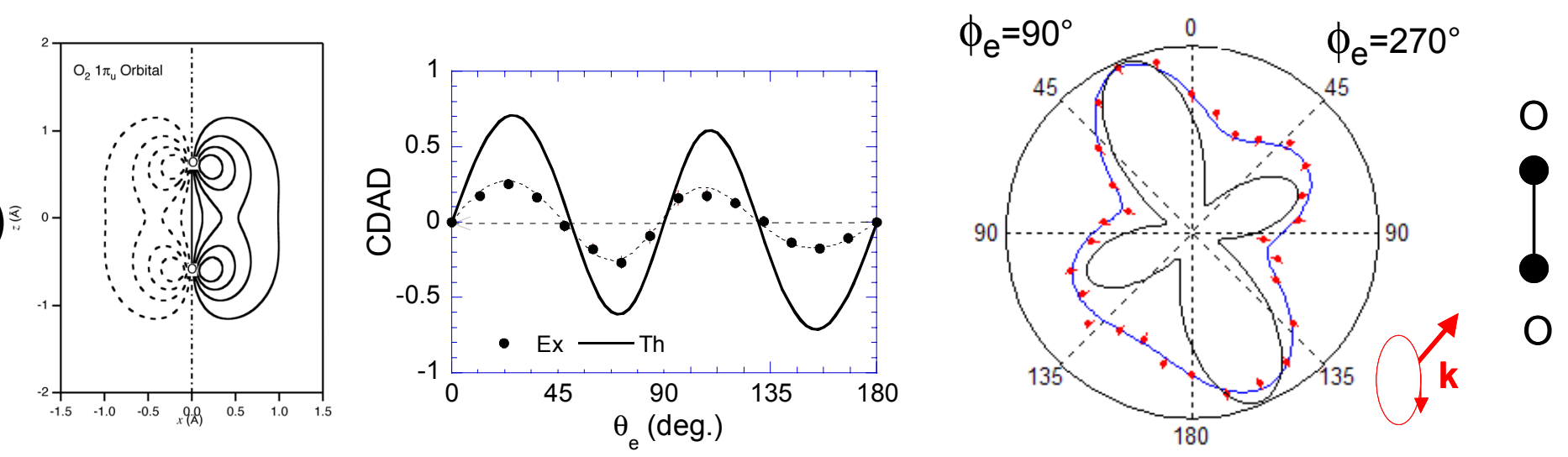

(b)
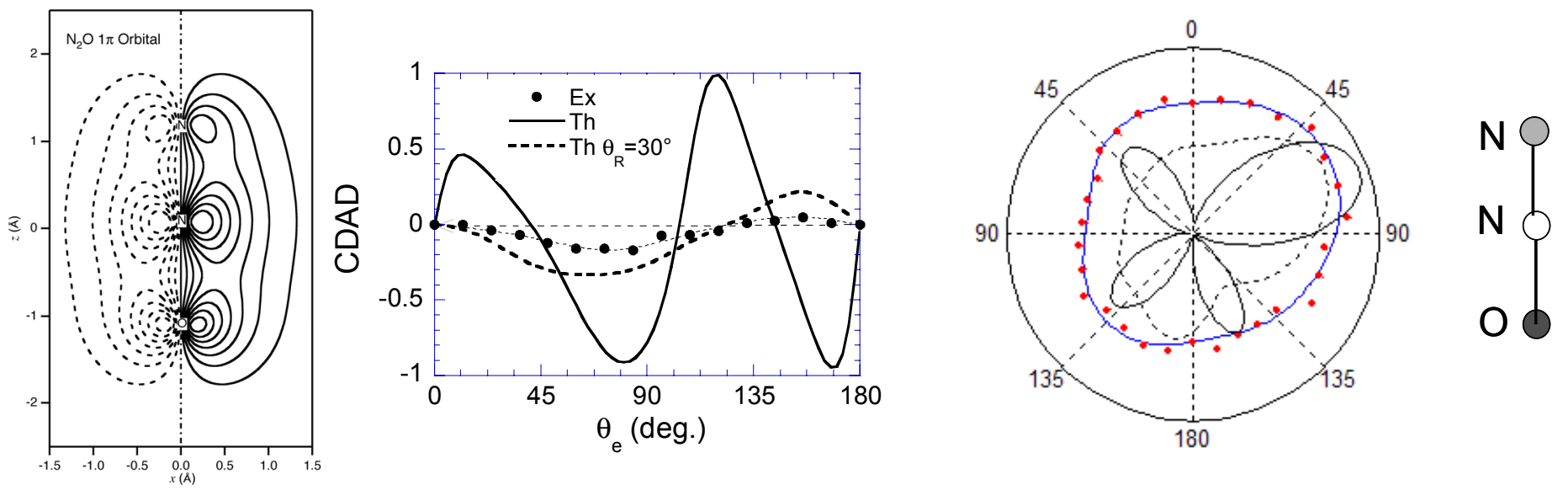

Figure 4 

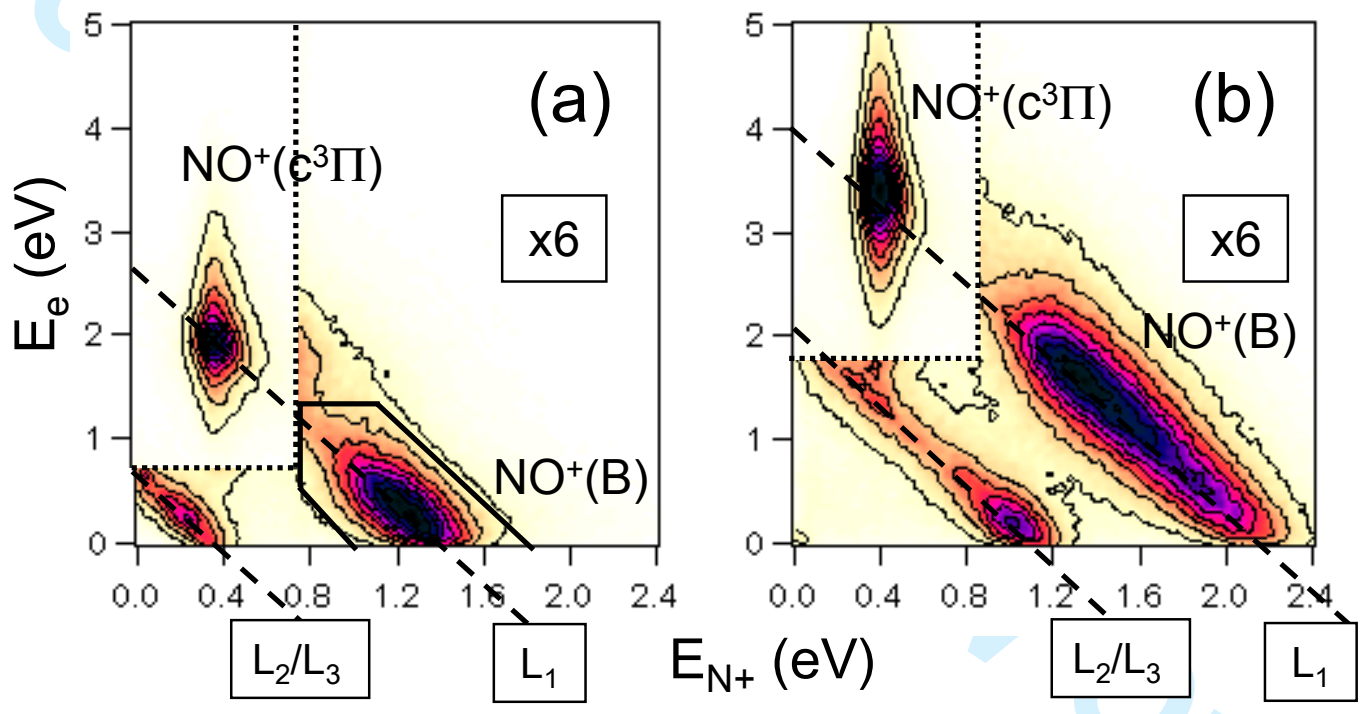

Figure 5 


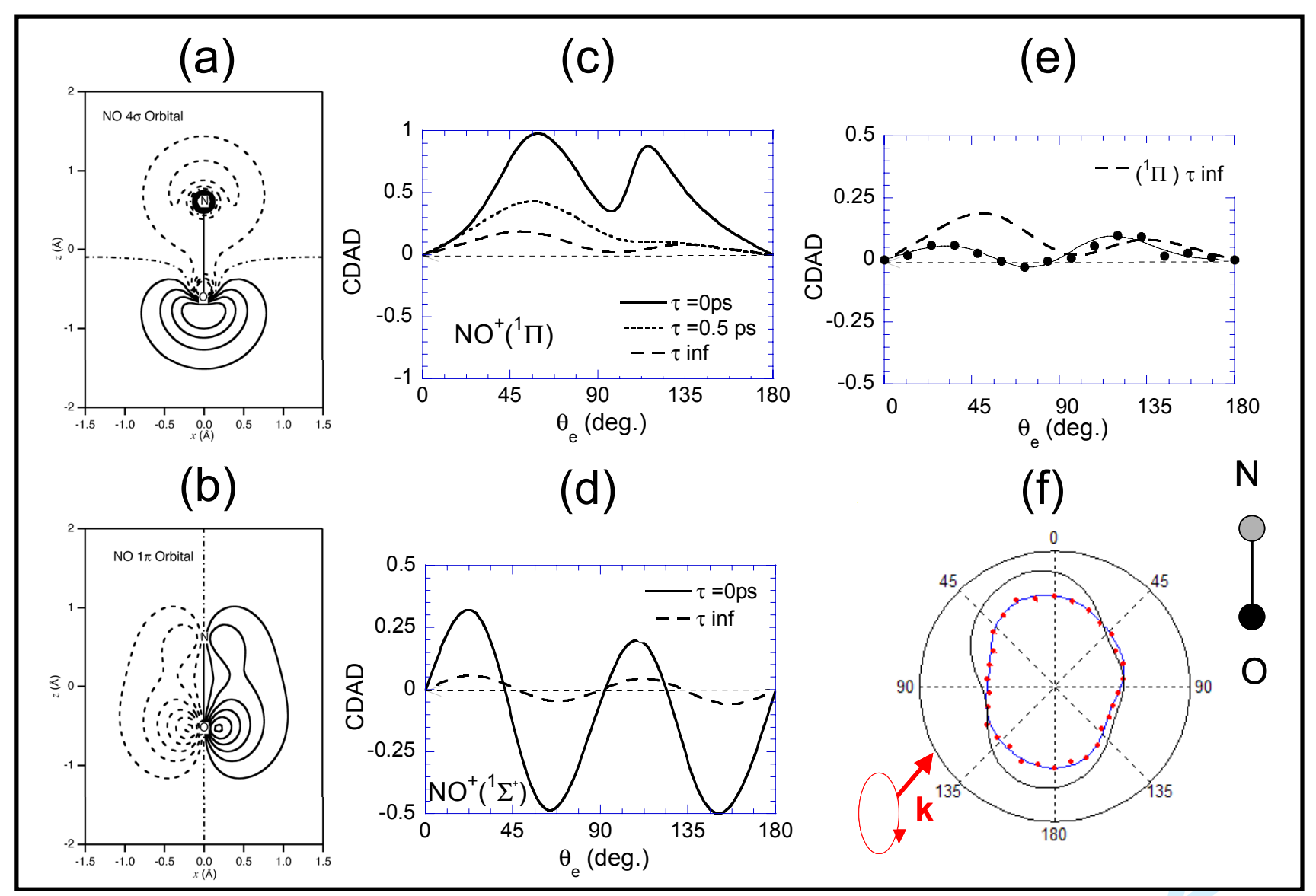

Figure 6 


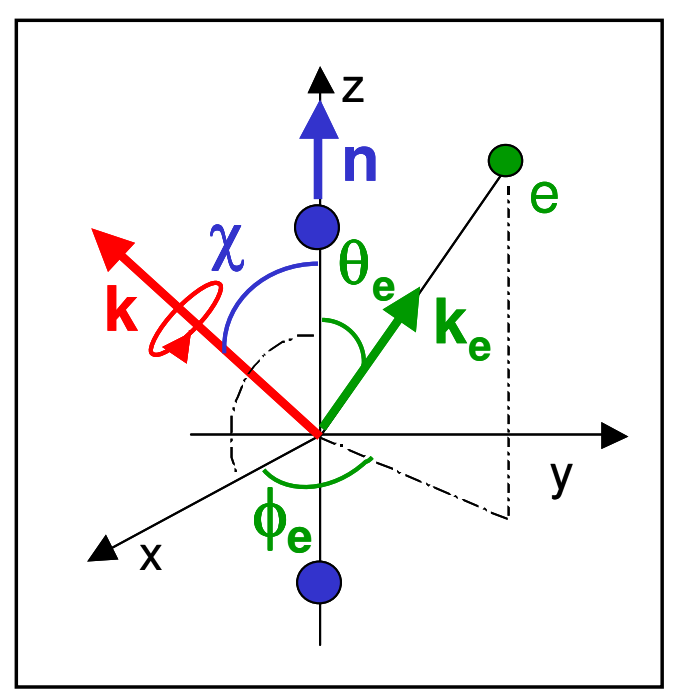

Figure 1 
(a)

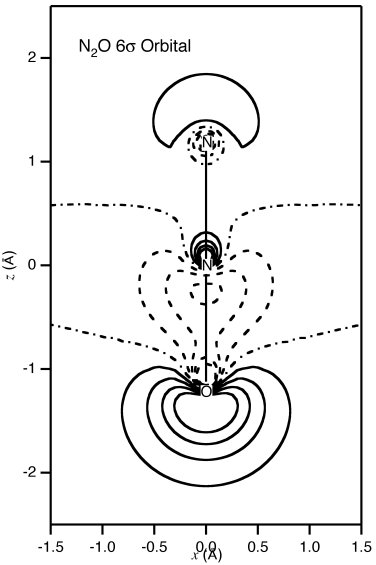

(b)

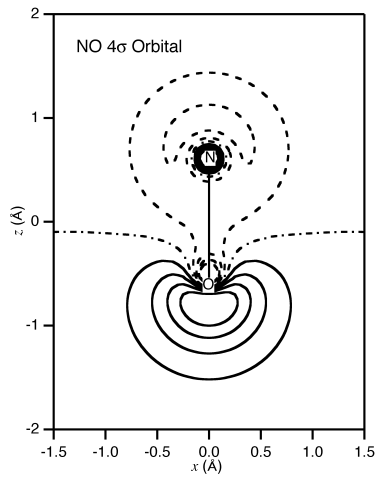

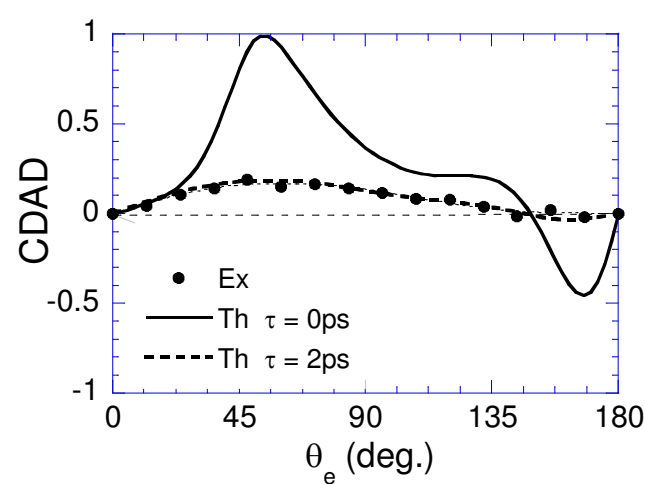
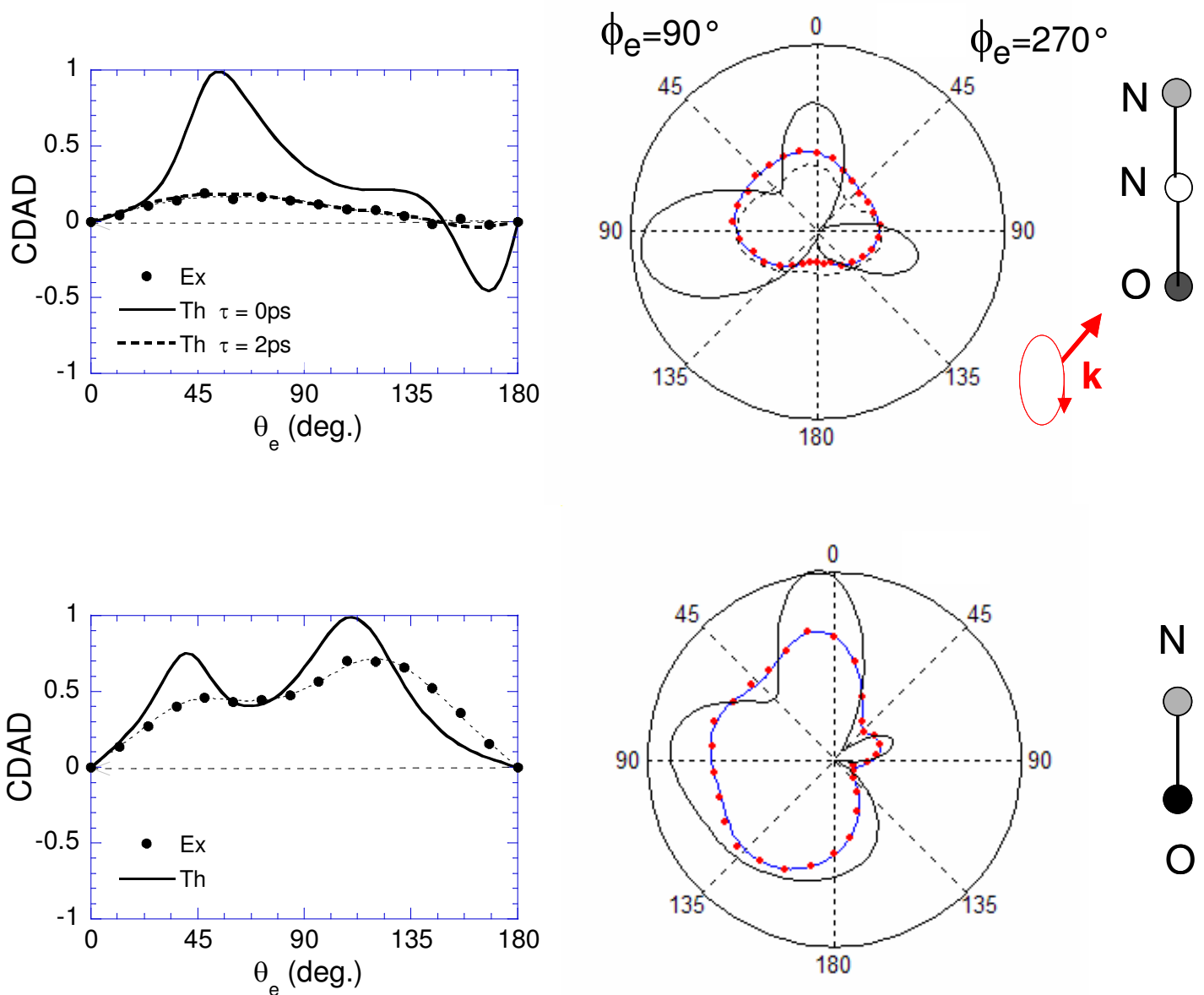

Figure 2

URL: http://mc.manuscriptcentral.com/tandf/tmph 


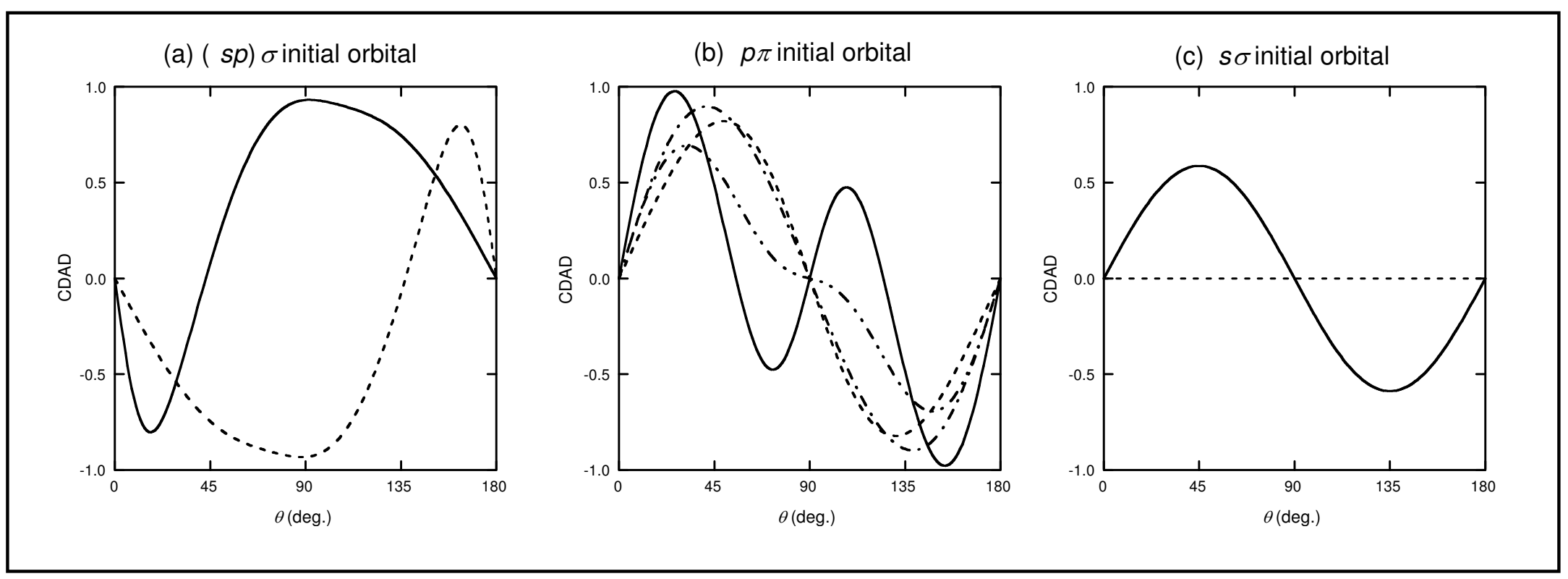

Figure 3

URL: http://mc.manuscriptcentral.com/tandf/tmph 


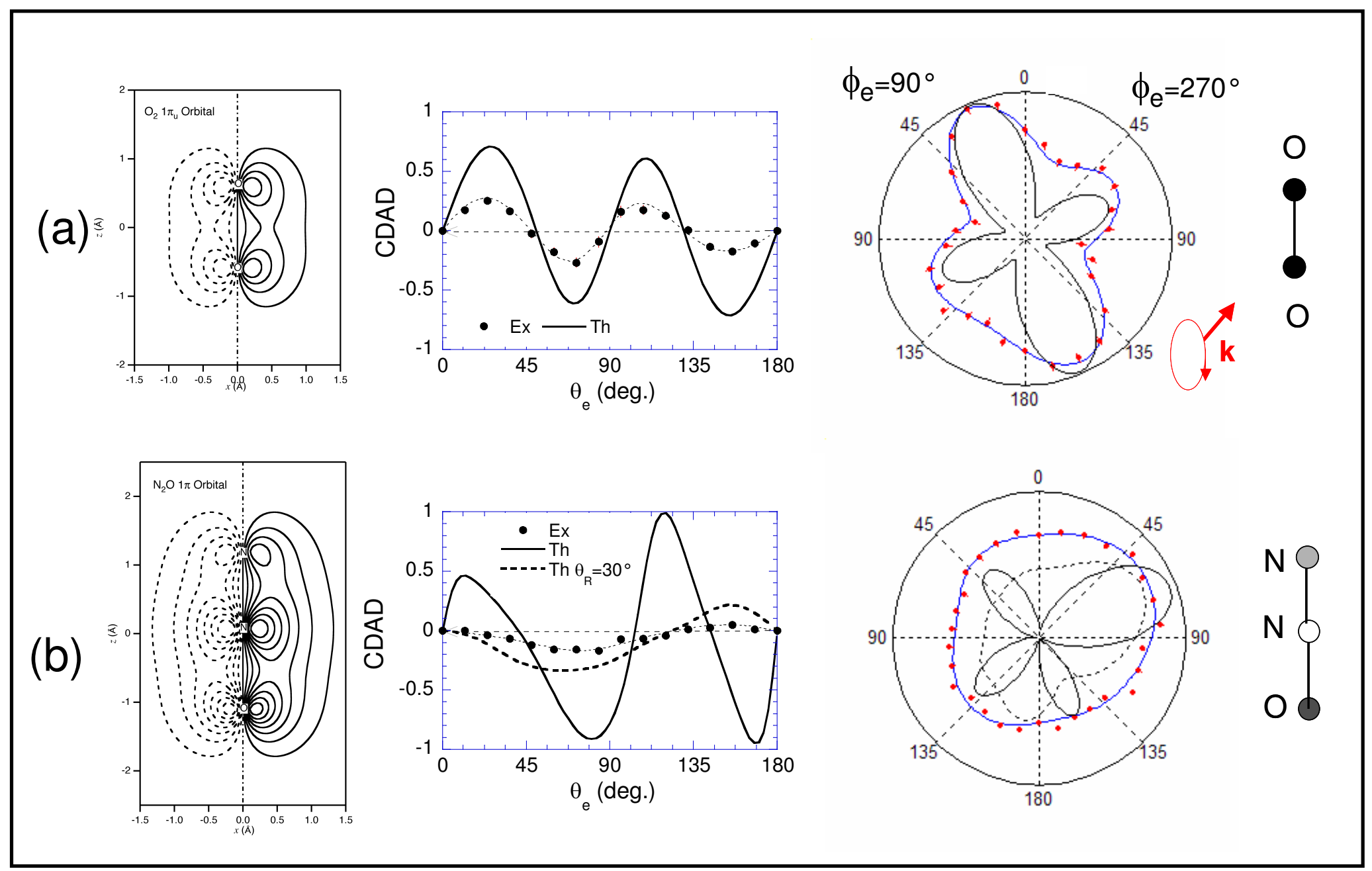

Figure 4

URL: http://mc.manuscriptcentral.com/tandf/tmph 

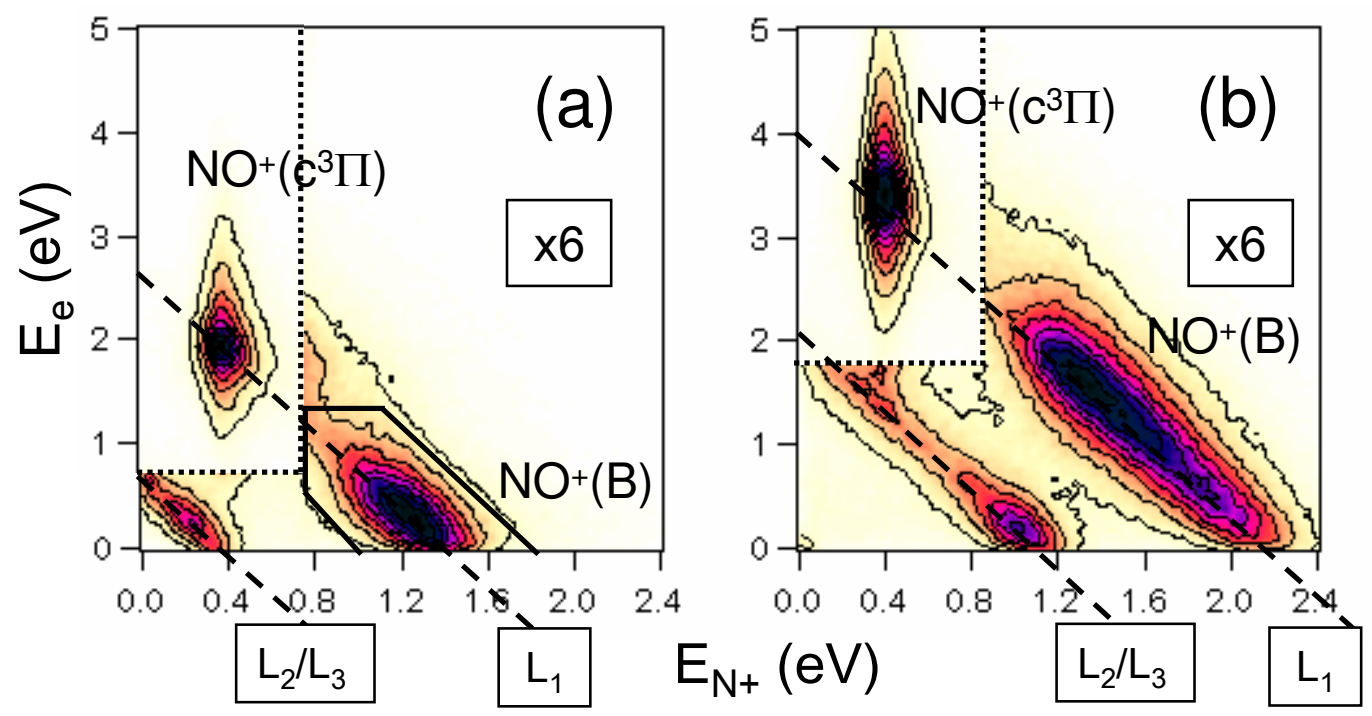

Figure 5

URL: http://mc.manuscriptcentral.com/tandf/tmph 


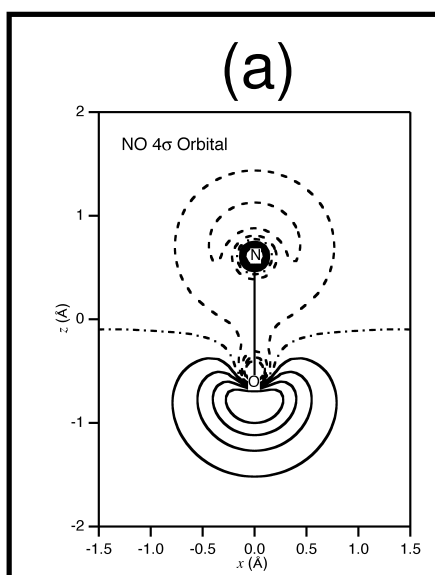

(b)

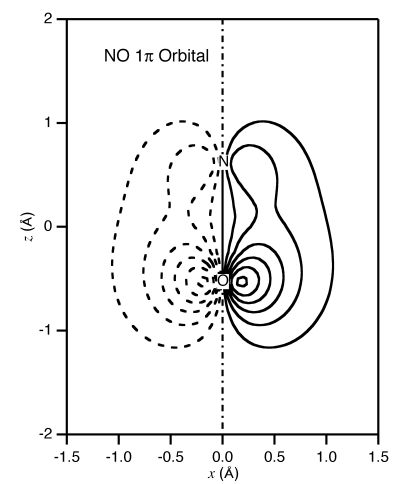

(c)

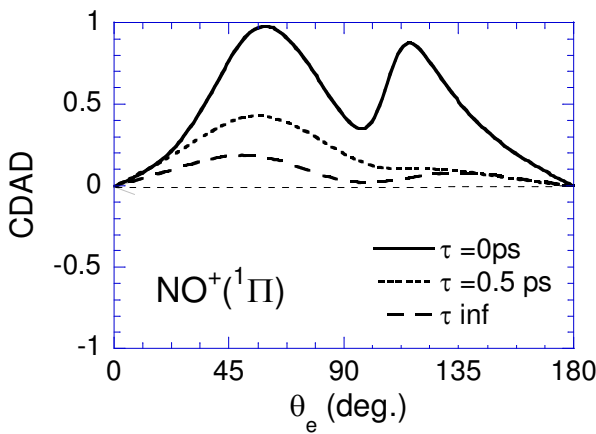

(d)

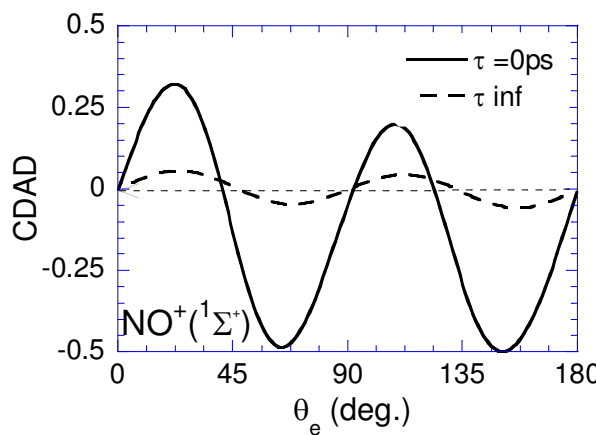

(e)

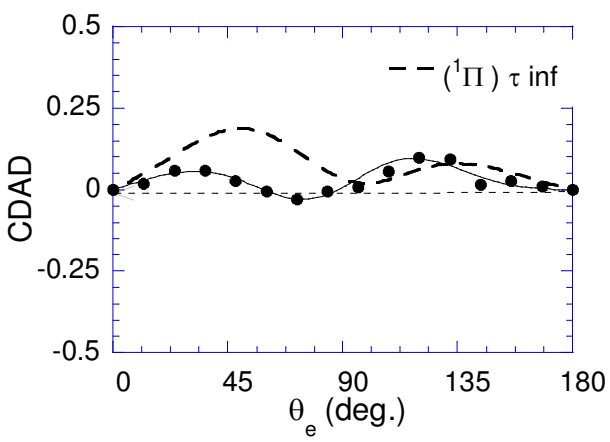

(f)

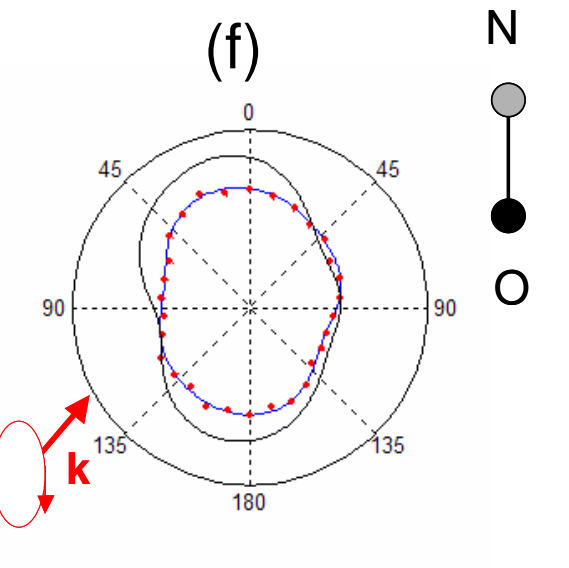

Figure 6 